\title{
RNA-Seq in 296 phased trios provides a high-resolution map of genomic imprinting
}

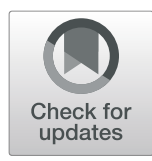

Bharati Jadhav ${ }^{1 \dagger}$, Ramin Monajemi ${ }^{2 *} \mathbb{D}$, Kristina K. Gagalova ${ }^{3}$, Daniel Ho ${ }^{1}$, Harmen H. M. Draisma ${ }^{2,4}$, Mark A. van de Wiel' ${ }^{5}$, Lude Franke ${ }^{6}$, Bastiaan T. Heijmans' ${ }^{2}$, Joyce van Meurs' ${ }^{7}$, Rick Jansen ${ }^{8}$, GoNL Consortium, BIOS Consortium, Peter A. C. 't Hoen ${ }^{4,9}$, Andrew J. Sharp ${ }^{1 *+}$ and Szymon M. Kiełbasa ${ }^{2^{*+}}$

\begin{abstract}
Background: Identification of imprinted genes, demonstrating a consistent preference towards the paternal or maternal allelic expression, is important for the understanding of gene expression regulation during embryonic development and of the molecular basis of developmental disorders with a parent-of-origin effect. Combining allelic analysis of RNA-Seq data with phased genotypes in family trios provides a powerful method to detect parent-of-origin biases in gene expression.

Results: We report findings in 296 family trios from two large studies: 165 lymphoblastoid cell lines from the 1000 Genomes Project and 131 blood samples from the Genome of the Netherlands (GoNL) participants. Based on parental haplotypes, we identified $>2.8$ million transcribed heterozygous SNVs phased for parental origin and developed a robust statistical framework for measuring allelic expression. We identified a total of 45 imprinted genes and one imprinted unannotated transcript, including multiple imprinted transcripts showing incomplete parental expression bias that was located adjacent to strongly imprinted genes. For example, PXDC1, a gene which lies adjacent to the paternally expressed gene $F A M 50 B$, shows a 2:1 paternal expression bias. Other imprinted genes had promoter regions that coincide with sites of parentally biased DNA methylation identified in the blood from uniparental disomy (UPD) samples, thus providing independent validation of our results. Using the stranded nature of the RNA-Seq data in lymphoblastoid cell lines, we identified multiple loci with overlapping sense/antisense transcripts, of which one is expressed paternally and the other maternally. Using a sliding window approach, we searched for imprinted expression across the entire genome, identifying a novel imprinted putative IncRNA in 13q21.2. Overall, we identified 7 transcripts showing parental bias in gene expression which were not reported in 4 other recent RNA-Seq studies of imprinting.
\end{abstract}

Conclusions: Our methods and data provide a robust and high-resolution map of imprinted gene expression in the human genome.

Keywords: Imprinting, Allele-specific expression, Bayesian analysis, Parent-of-origin, Phased genotypes

\footnotetext{
* Correspondence: r.monajemi@lumc.nl; andrew.sharp@mssm.edu; smkielbasa@lumc.nl

${ }^{\dagger}$ Bharati Jadhav, Ramin Monajemi, Andrew J. Sharp and Szymon M. Kiełbasa contributed equally to this work.

${ }^{2}$ Department of Biomedical Data Sciences, Leiden University Medical Center, Einthovenweg 20, 2333 ZC Leiden, the Netherlands

${ }^{1}$ Department of Genetics and Genomic Sciences, Hess Center for Science and Medicine, Mount Sinai School of Medicine, 1470 Madison Avenue, Room 8-116, Box 1498, New York, NY 10029, USA

Full list of author information is available at the end of the article
}

(c) The Author(s). 2019 Open Access This article is distributed under the terms of the Creative Commons Attribution 4.0 International License (http://creativecommons.org/licenses/by/4.0/), which permits unrestricted use, distribution, and reproduction in any medium, provided you give appropriate credit to the original author(s) and the source, provide a link to the Creative Commons license, and indicate if changes were made. The Creative Commons Public Domain Dedication waiver (http://creativecommons.org/publicdomain/zero/1.0/) applies to the data made available in this article, unless otherwise stated. 


\section{Background}

Genomic imprinting is a special case of mono-allelic expression where genes are expressed in a parent-oforigin (PofO)-specific manner. Although several hypotheses exist to explain why genomic imprinting occurs, the parental conflict hypothesis [1] posits that imprinted genes evolved from a parental battle between males and females to influence the allocation of maternal resources to offspring. This type of mono-allelic expression can be observed in mammals at different developmental stages and is dependent on stage, cell, and tissue type.

Genomic imprinting plays a vital role in normal development, and errors of imprinting can underlie developmental disorders and contribute to certain cancers [2]. Imprinting significantly influences the development of cell lineages, prenatal growth, normal brain function, and metabolism [3]. Any disruption to the imprinted genes can lead to disturbed gene function and can have a deleterious effect on health. If such disruption happens at imprinted loci, it can result in imprinting disorders such as Beckwith-Wiedemann, Silver-Russell [4], Prader-Willi, and Angelman syndromes [5]; transient neonatal diabetes [6], and cancer. Wilms' tumor, colorectal cancer, and hepatoblastoma are few examples of cancer caused due to aberrant imprinting in the IGF2 gene $[7,8]$.

There are many screening methods developed and applied to discover imprinted genes such as DNA methylation, histone modification, and gene expression assays. RNA sequencing (RNA-Seq) is the most direct and comprehensive way to identify imprinted genes as it allows for quantifying relative expression of the maternal and paternal alleles (allele-specific expression or ASE) at all heterozygous sites with sufficient coverage. However, the technology is subject to several technical biases resulting in potential false positives [9]. The reference bias, caused by additional penalties in the alignment for non-reference alleles, is the most prominent of these biases [10]. Moreover, the availability of additional DNA genotype information is essential because the heterozygous sites may appear as homozygous in the RNA because of mono-allelic expression of the imprinted genes. Typically, such studies are performed without allelic inheritance information and make use of the bimodal distribution of the expression at heterozygous sites $[11,12]$. This type of analysis lacks the ability to identify directionality of parental bias (i.e., assessing maternal versus paternal imprinting). Adding PofO information allows robust determination of maternal versus paternal allelespecific expression, particularly in the case of incomplete imprinting (slight bias towards the paternal or maternal allele), where bimodality in the distribution is difficult to assess. The use of PofO information is straightforward in mouse studies where reciprocal cross design is often used to identify maternal/paternal gene expression and imprinted genes [13-15]. However, in humans, assignment of parental origin requires genotype data from multiple generations. Until recently, such studies have been limited to relatively small numbers of family pedigrees [16, 17], although analyses of imprinting in much larger pedigrees have been reported recently $[18,19]$.

Here, we present a robust genome-wide approach to find PofO-specific gene expression and identify the signature of imprinted genes at heterozygous sites using phased DNA genotypes from parent-offspring trios and RNA-Seq data aggregated at the gene level. Our method is applied to two large-scale studies with a total of 296 trios: 165 trios from the HapMap/1000 Genomes Projects with RNA-Seq data from lymphoblastoid cell lines (LCLs) and 131 trios from the Genome-of-theNetherlands [20]. We focus on the identification of genes and transcripts that are consistently imprinted in the population, detecting both complete imprinting (exclusive expression of the paternal or maternal allele) and incomplete imprinting (bias in expression towards the maternal or paternal allele).

\section{Results}

We tested for imprinted gene expression using allelespecific RNA-Seq analysis of 296 parent-offspring trios derived from two independent cohorts: (i) 165 LCLs collected as part of the HapMap Project and (ii) 131 whole blood (WB) samples studied by the Genome of the Netherlands (GoNL) Consortium. In each cohort, we used phased genotypes to compute the relative expression from the maternal and paternal alleles in RNA-Seq reads at expressed heterozygous single nucleotide variants (SNVs). We analyzed 23,003 Gencode genes which had at least one heterozygous SNV with $\geq 1$ overlapping RNA-Seq reads in $>10 \%$ of the samples and summed the paternal and maternal counts for all heterozygous SNVs contained in a gene, irrespective of their exonic or intronic nature. The inclusion of intronic SNVs increased the power of our test considerably despite their low individual coverage, as there were generally many more informative intronic than exonic SNVs. We applied two statistical tests to check for consistent parental expression bias of autosomal genes within the populations. The rationale for using two statistical tests, Wilcoxon signed-rank (WSR) test and ShrinkBayes (SB), is their differences in power and falsepositive rate in case of low numbers of informative individuals and low expression. More details are given in Additional files 1 and 2 .

Quantile-quantile plots showed a clear excess of genes with highly significant observed $p$ values above the null expectation with both statistical tests and cohorts, indicating strong evidence for imprinting. Furthermore, there was no evidence of genomic inflation in our study, with all values of $\lambda$ between 0.9999 and 1.02 (Fig. 1). To 


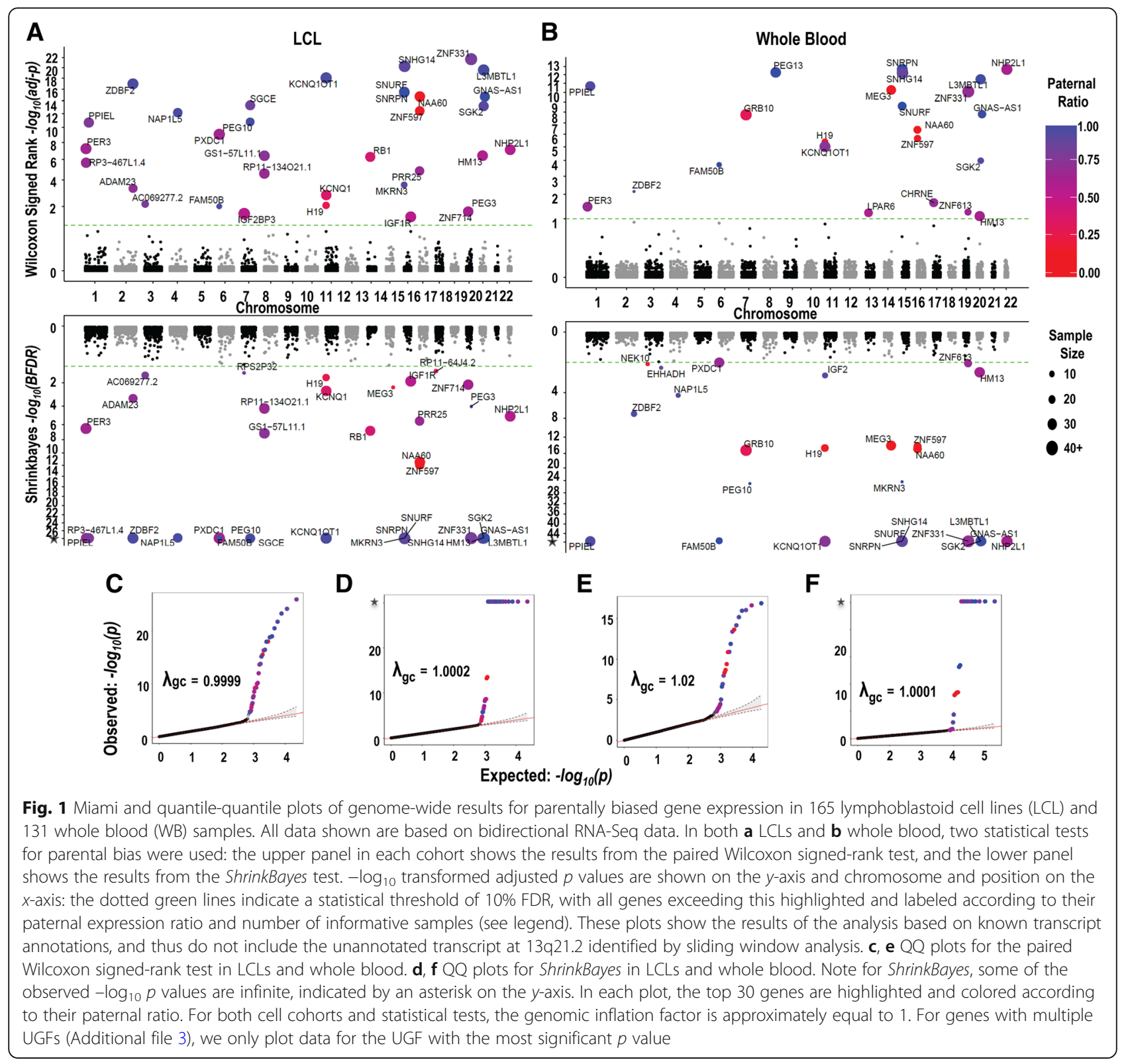

increase the resolution and avoid confounding in cases where multiple different transcripts overlapped each other, we used unique gene fragment (UGF; see the "Methods" section) annotation as the basic genomic units. Overall a total of 78 UGFs across the two populations showed significant evidence of imprinting (Additional files 3 and 4): 66 in LCLs and 43 in WB. However, the presence of overlapping transcripts, some of which were split into multiple separate annotations by our use of UGFs, created redundancy in this list. After removal of these redundancies, we further manually curated signals to (i) assign signals of imprinted expression to the gene annotation which showed the best consistency with the strand and location of data, (ii) remove transcripts where biased expression was driven by outlier samples with extreme read depth, and (iii) at loci containing multiple overlapping gene annotations, to avoid inflating the number of reported genes, we removed anonymous transcripts which appeared to represent partial gene fragments (see comments in Additional file 3). This identified a total of 45 imprinted genes across the two cohorts: 38 in LCLs and 31 in WB, with 23 identified in both populations (Fig. 1, Additional file 5: Fig. A). The paternal ratios for each of these genes in each individual are plotted in Fig. 2.

For each dataset, we classified genes as high confidence if significant (10\% FDR) in both statistical tests (34 in 


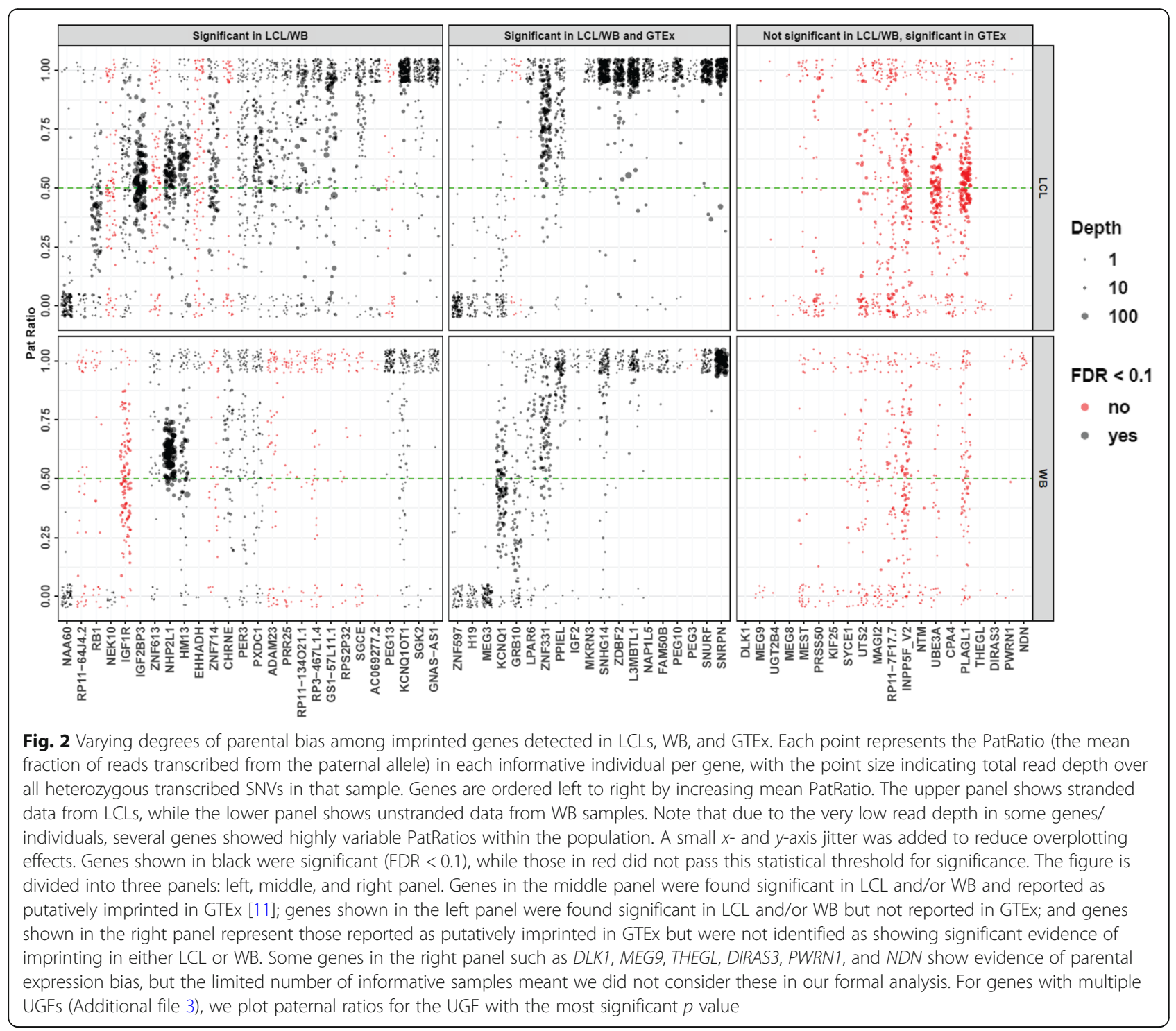

LCLs and 19 in WB). Genes were scored as low confidence if identified as significant by a single statistical test (4 in LCLs and 11 in WB) (see Additional file 5: Fig. B and C). At 10\% FDR using the paired sample Wilcoxon signed-rank (WSR) test, we found 36 and 24 significant genes in LCLs and WB, respectively. With ShrinkBayes (SB), we found 37 and 27 significant genes in LCLs and WB, respectively, at 10\% FDR (Tables 1 and 2).

We compared the 45 imprinted genes in our dataset with those from two studies of imprinting in the Genotype-Tissue Expression (GTEx) Project [11, 12], showing that 28 were also identified as imprinted in GTEx, with one additional gene identified as "putatively imprinted" (Fig. 2, Additional file 6). In several cases, genes identified as imprinted in the GTEx cohort that we failed to replicate (e.g., DLK1, MEG9, THEGL,
DIRAS3, PWRN1, and NDN) showed clear evidence of parental expression bias in our raw data, but the limited number of informative samples in our study populations meant we did not consider these in our formal analysis (Fig. 2). In all cases, we observed consistent directionality of parental bias between the two studies. Furthermore, comparison with a recent study of imprinting in a large Icelandic cohort also showed strong concordance, with 38 of the genes we identified as imprinted also observed by [19] (Additional file 6).

Using only female samples, we searched for signals of imprinting on the $\mathrm{X}$ chromosome. We first estimated $\mathrm{X}$ chromosome inactivation ratios (XCIRs) in each female, removing those samples that showed highly biased XCIR ( $>80 \%$ silencing of one $\mathrm{X}$ chromosome), and then normalized allelic read counts for X-linked genes in each sample 
Table 1 High-confidence imprinted genes identified in LCLs and whole blood

\begin{tabular}{|c|c|c|c|c|c|c|c|c|c|}
\hline & Gene name & Chr & $\begin{array}{l}\text { Start } \\
\text { (hg19) }\end{array}$ & $\begin{array}{l}\text { End } \\
\text { (hg19) }\end{array}$ & $\begin{array}{l}\text { Cytogenetic } \\
\text { band }\end{array}$ & Strand & $\begin{array}{l}\text { Pat ratio } \\
\left(\mathrm{LCLL}_{S} / \mathrm{LCL} / \mathrm{WB}\right)\end{array}$ & $\begin{array}{l}\text { Preferentially } \\
\text { expressed allele }\end{array}$ & $\begin{array}{l}\text { Confidence } \\
\text { (LCL/WB) }\end{array}$ \\
\hline 1 & PER3 & 1 & 7844380 & 7905237 & p36.23 & + & $0.61 / 0.65 / 0.68$ & Paternal & $\mathrm{HC} / \mathrm{LC}$ \\
\hline 2 & RP3-467L 1.4 & 1 & 7870302 & 7887402 & p36.23 & - & $0.81 / 0.69 / 0.62$ & Paternal & $\mathrm{HCl}-$ \\
\hline 3 & PPIEL & 1 & 39997510 & 40024379 & p34.3 & - & 0.78/0.78/0.90 & Paternal & $\mathrm{HC} / \mathrm{HC}$ \\
\hline 4 & ZDBF2 & 2 & 207139387 & 207179148 & q33.3 & + & 0.94/0.94/0.95 & Paternal & $\mathrm{HC} / \mathrm{HC}$ \\
\hline 5 & ADAM23 & 2 & 207308263 & 207485851 & q33.3 & + & $0.71 / 0.70 / 0.63$ & Paternal & $\mathrm{HCl}-$ \\
\hline 6 & AC069277.2 & 3 & 6532166 & 6777816 & p26.1 & + & 0.79/0.79/0.80 & Paternal & $\mathrm{HCl}-$ \\
\hline 7 & NAP1L5 & 4 & 89617066 & 89619386 & q22.1 & - & 0.97/0.95/0.93 & Paternal & $\mathrm{HC} / \mathrm{LC}$ \\
\hline 8 & PXDC1 & 6 & 3722848 & 3752260 & p25.2 & - & $0.65 / 0.64 / 0.68$ & Paternal & $\mathrm{HC} / \mathrm{LC}$ \\
\hline 9 & FAM50B & 6 & 3849620 & 3851551 & p25.2 & + & 0.94/0.94/1.00 & Paternal & $\mathrm{HC} / \mathrm{HC}$ \\
\hline 10 & GRB10 & 7 & 50657760 & 50861159 & p12.1 & - & $0.57 / 0.57 / 0.29$ & Maternal & $-/ \mathrm{HC}$ \\
\hline 11 & SGCE & 7 & 94214542 & 94285521 & $\mathrm{q} 21.3$ & - & 0.83/0.83/0.53 & Paternal & $\mathrm{HCl}-$ \\
\hline 12 & PEG10 & 7 & 94285637 & 94299007 & q21.3 & + & 0.97/0.97/1.00 & Paternal & $\mathrm{HC} / \mathrm{LC}$ \\
\hline 13 & RP11-134021.1 & 8 & 2523591 & 2585991 & p23.2 & - & 0.70/0.68/0.78 & Paternal & $\mathrm{HCl}-$ \\
\hline 14 & GS1-57L11.1 & 8 & 2584858 & 2680004 & p23.2 & + & 0.73/0.73/0.91 & Paternal & $\mathrm{HCl}-$ \\
\hline 15 & H19 & 11 & 2016406 & 2022700 & p15.5 & - & $0.10 / 0.26 / 0.00$ & Maternal & $\mathrm{HC} / \mathrm{HC}$ \\
\hline 16 & KCNQ1 & 11 & 2465914 & 2870339 & p15.5 & + & $0.18 / 0.36 / 0.46$ & Maternal & $\mathrm{HC} / \mathrm{HC}$ \\
\hline 17 & KCNQ1OT1 & 11 & 2629558 & 2721224 & p15.5 & - & 0.96/0.94/0.74 & Paternal & $\mathrm{HC} / \mathrm{HC}$ \\
\hline 18 & $R B 1$ & 13 & 48877887 & 49056122 & $\mathrm{q} 14.2$ & + & $0.39 / 0.39 / 0.54$ & Maternal & $\mathrm{HC} /-$ \\
\hline 19 & LPAR6 & 13 & 48963707 & 49018840 & q14.2 & - & 0.87/0.39/0.60 & Paternal & $\mathrm{HC} / \mathrm{LC}$ \\
\hline 20 & MEG3 & 14 & 101245747 & 101327368 & q32.2 & + & $0.21 / 0.24 / 0.02$ & Maternal & $\mathrm{LC} / \mathrm{HC}$ \\
\hline 21 & MKRN3 & 15 & 23810454 & 23873064 & q11.2 & + & 0.90/0.90/1.00 & Paternal & HC/LC \\
\hline 22 & SNRPN & 15 & 25068794 & 25223870 & q11.2 & + & 0.98/0.98/1.00 & Paternal & $\mathrm{HC} / \mathrm{HC}$ \\
\hline 23 & SNURF & 15 & 25200181 & 25245423 & $\mathrm{q} 11.2$ & + & 0.98/0.98/1.00 & Paternal & $\mathrm{HC} / \mathrm{HC}$ \\
\hline 24 & SNHG14 & 15 & 25223730 & 25664609 & q11.2 & + & 0.98/0.89/0.88 & Paternal & $\mathrm{HC} / \mathrm{HC}$ \\
\hline 25 & IGF1R & 15 & 99192200 & 99507759 & q26.3 & + & $0.56 / 0.56 / 0.50$ & Paternal & $\mathrm{HCl}-$ \\
\hline 26 & PRR25 & 16 & 855443 & 863861 & p13.3 & + & 0.69/0.67/0.66 & Paternal & $\mathrm{HCl}-$ \\
\hline 27 & ZNF597 & 16 & 3486104 & 3493542 & p13.3 & - & $0.04 / 0.06 / 0.05$ & Maternal & $\mathrm{HC} / \mathrm{HC}$ \\
\hline 28 & NAA60 & 16 & 3493611 & 3536963 & p13.3 & + & 0.06/0.05/0.03 & Maternal & $\mathrm{HC} / \mathrm{HC}$ \\
\hline 29 & ZNF714 & 19 & 21264965 & 21308073 & p12 & + & $0.62 / 0.62 / 0.63$ & Paternal & $\mathrm{HCl}-$ \\
\hline 30 & ZNF613 & 19 & 52430400 & 52452012 & q13.41 & + & $0.50 / 0.50 / 0.67$ & Paternal & $-/ H C$ \\
\hline 31 & ZNF331 & 19 & 54024235 & 54083523 & q13.42 & + & 0.81/0.81/0.70 & Paternal & $\mathrm{HC} / \mathrm{HC}$ \\
\hline 32 & PEG3 & 19 & 57321445 & 57352096 & q13.43 & - & 0.98/0.98/1.00 & Paternal & $\mathrm{HC} /-$ \\
\hline 33 & HM13 & 20 & 30102231 & 30157370 & $q 11.21$ & + & $0.57 / 0.58 / 0.63$ & Paternal & $\mathrm{HC} / \mathrm{HC}$ \\
\hline 34 & L3MBTL 1 & 20 & 42136320 & 42179590 & q13.12 & + & 0.96/0.96/0.97 & Paternal & $\mathrm{HC} / \mathrm{HC}$ \\
\hline 35 & SGK2 & 20 & 42187608 & 42216877 & q13.12 & + & $0.92 / 0.91 / 0.93$ & Paternal & $\mathrm{HC} / \mathrm{HC}$ \\
\hline 36 & GNAS-AS1 & 20 & 57393974 & 57425958 & q13.32 & - & 0.96/0.96/0.98 & Paternal & $\mathrm{HC} / \mathrm{HC}$ \\
\hline 37 & NHP2L1 & 22 & 42069934 & 42086508 & q13.2 & - & $0.57 / 0.57 / 0.62$ & Paternal & $\mathrm{HC} / \mathrm{HC}$ \\
\hline
\end{tabular}

High-confidence imprinted genes were classified as those transcripts showing significant evidence of parental expression bias (at 10\% FDR) by both statistical tests used in at least one of the two cohorts studied. $\mathrm{LCL}_{s}$ and $\mathrm{LCL}_{\mathrm{u}}$ indicate the results from $\mathrm{LCL}$ stranded and unstranded data, respectively. For genes with multiple UGFs (Additional file 3), we report paternal ratios for the UGF with the most significant $p$ value

based on their XCIR. Analyses of these data resulted in one gene showing putative significant parental bias in LCLs (RNA28S5) and one gene in WB (ARSD). However, both were discounted as false-positive signals due to clear reference bias in both cases (Additional file 7: Fig. A-E).

\section{Exclusion of potential confounders}

It has been reported that LCLs can sometimes undergo clonal expansion, which in turn can lead to elevated rates of mono-allelic expression [21]. As this has the potential to create artifacts that might resemble imprinting, 
Table 2 Low-confidence imprinted genes identified in either LCLs or whole blood

\begin{tabular}{|c|c|c|c|c|c|c|c|c|c|}
\hline & Gene name & Chr & $\begin{array}{l}\text { Start } \\
\text { (hg19) }\end{array}$ & $\begin{array}{l}\text { End } \\
\text { (hg19) }\end{array}$ & $\begin{array}{l}\text { Cytogenetic } \\
\text { band }\end{array}$ & Strand & $\begin{array}{l}\text { Pat ratio } \\
\left(\mathrm{LCL}_{S} / \mathrm{LCL} / \mathrm{WB}\right)\end{array}$ & $\begin{array}{l}\text { Preferentially } \\
\text { expressed allele }\end{array}$ & $\begin{array}{l}\text { Confidence } \\
\text { (LCL/WB) } \\
\end{array}$ \\
\hline 1 & NEK10 & 3 & 27151576 & 27410951 & p24.1 & - & $0.48 / 0.48 / 0.18$ & Maternal & $-/ L C$ \\
\hline 2 & EHHADH & 3 & 184908412 & 184999778 & $\mathrm{q} 27.2$ & - & $0.58 / 0.58 / 0.88$ & Paternal & $-/ L C$ \\
\hline 3 & IGF2BP3 & 7 & 23349828 & 23510086 & p15.3 & - & $0.54 / 0.54 / 1.00$ & Paternal & $\mathrm{LC} /-$ \\
\hline 4 & RPS2P32 & 7 & 23530092 & 23530983 & p15.3 & + & 0.88/0.79/0.64 & Paternal & $\mathrm{LC} /-$ \\
\hline 5 & PEG13 & 8 & 141104993 & 141110634 & $\mathrm{q} 24.3$ & - & $0.47 / 0.47 / 0.99$ & Paternal & $-/ L C$ \\
\hline 6 & IGF2 & 11 & 2150342 & 2170833 & p15.5 & - & NA/ NA/0.89 & Paternal & $-/ \mathrm{LC}$ \\
\hline 7 & (unannotated transcript) & 13 & 60794418 & 60853802 & $\mathrm{q} 21.2$ & + & NA/0.86/NA & Paternal & LC/- \\
\hline 8 & $R P 11-64 J 4.2$ & 17 & 3182069 & 3289633 & p13.3 & - & $0.27 / 0.30 / 0.49$ & Maternal & $\mathrm{LC} /-$ \\
\hline 9 & CHRNE & 17 & 4801069 & 4806369 & p13.2 & - & $0.56 / 0.59 / 0.70$ & Paternal & $-/ L C$ \\
\hline
\end{tabular}

Low-confidence imprinted genes were classified as those transcripts showing significant evidence of parental expression bias (at 10\% FDR) by just one statistical test in one of the two cohort studied. $\mathrm{LCL}_{s}$ and $\mathrm{LCL}_{u}$ indicate the results from $\mathrm{LCL}$ stranded and unstranded data, respectively. For genes with multiple UGFs (Additional file 3), we report paternal ratios for the UGF with the most significant $p$ value

we utilized the XCIRs we defined in females to identify and exclude LCLs with possible clonality. Focusing only on those female LCLs without skewed XCIR (XCIRs between 0.2 and $0.8, n=45$ ), we repeated the WSR test for imprinting on the 56 autosomal UGFs that had informative SNVs in at least 5 of these non-clonal LCLs. Even with this markedly reduced sample size, every gene tested showed very similar paternal ratios to those obtained in the full cohort of 165 LCLs, with 36 of the 38 (95\%) genes that we report as being imprinted in LCLs achieving at least nominal significance for unequal expression of the two parental alleles (Additional file 8). Thus, we were able to exclude the possibility that artifacts due to clonality in the LCLs we studied were driving our results.

Other studies have indicated that DNA methylation can become altered during the transformation and extended culture of LCLs, raising the possibility that this might create artifacts in our LCL cohort. To assess the stability of DNA methylation at imprinted loci in LCLs, we compared published datasets of DNA methylation in LCLs and blood and compared these with methylation profiles in samples with genome-wide uniparental disomy that shows loss of imprinting (Additional file 9). This analysis showed that there was no evidence for systematic loss of imprinting in LCLs and that methylation at the differentially methylated regions of imprinted loci is broadly similar between the blood and LCLs.

\section{Incomplete imprinting often clusters adjacent to strongly imprinted genes}

Most previous studies have identified imprinted genes based on the complete silencing of one parental allele. However, our large population sample and the quantitative nature of our assay identified several genes with biallelic expression, but which showed a significant bias for increased expression of one of the two parental alleles (Fig. 2). In many cases, these incompletely imprinted genes occurred in close proximity to previously known imprinted genes that show mono-allelic expression. For example, we identified $P X D C 1$, which lies $\sim 100 \mathrm{~kb}$ distal to the known imprinted FAM $50 B$ at $6 \mathrm{p} 25.2$, as showing a $2: 1$ paternal expression bias (PXDC1, paternal ratio of 0.65 and 0.68 in LCL and WB, respectively) (Fig. 3), in line with recently published studies $[18,19]$. Similarly, ADAM23, which lies $\sim 130 \mathrm{~kb}$ distal to $Z D B F 2$ at $2 \mathrm{q} 33.3$, also exhibits $\sim 2$-fold overexpression from the paternal allele (ADAM23, paternal ratio of 0.71 in $\mathrm{LCL}$ ), consistent with previous reports in both humans and mice $[15,16,22]$. Overall, we identified 11 clusters of imprinted genes (defined here as two or more imprinted genes separated by $<500 \mathrm{~kb}$ ), with 25 of the 45 imprinted genes we report located in these clusters. Using published datasets of imprinted DNA methylation [19, 23, 24], we observed that in several cases, genes with incomplete imprinting lie in close proximity to the regions with parental-specific methylation marks, providing independent support for imprinting at these loci (Additional file 10). Notable examples include PRR25 (paternal ratio $=0.69$ in LCLs) and the overlapping transcripts PER3/RP3-467L1.4 (paternal ratio $=0.61$ and 0.81 , respectively, in LCLs, shown in Additional file 11). A recent study [19] identified that both PRR25 and PER3 overlap CpG islands showing preferential maternal methylation. While Zink et al. did identify PER3 and RP3-467L1.4 as showing parental expression bias, it was not reported that the PRR25 gene itself was imprinted. Thus, our data suggest PRR25 is a novel incompletely imprinted gene.

To systematically investigate whether weaker imprinting localizes around strongly imprinted genes, we used data from a sliding window analysis across the genome in LCLs (detailed below) to test for enrichment of parental expression bias around known imprinted genes. Here, 


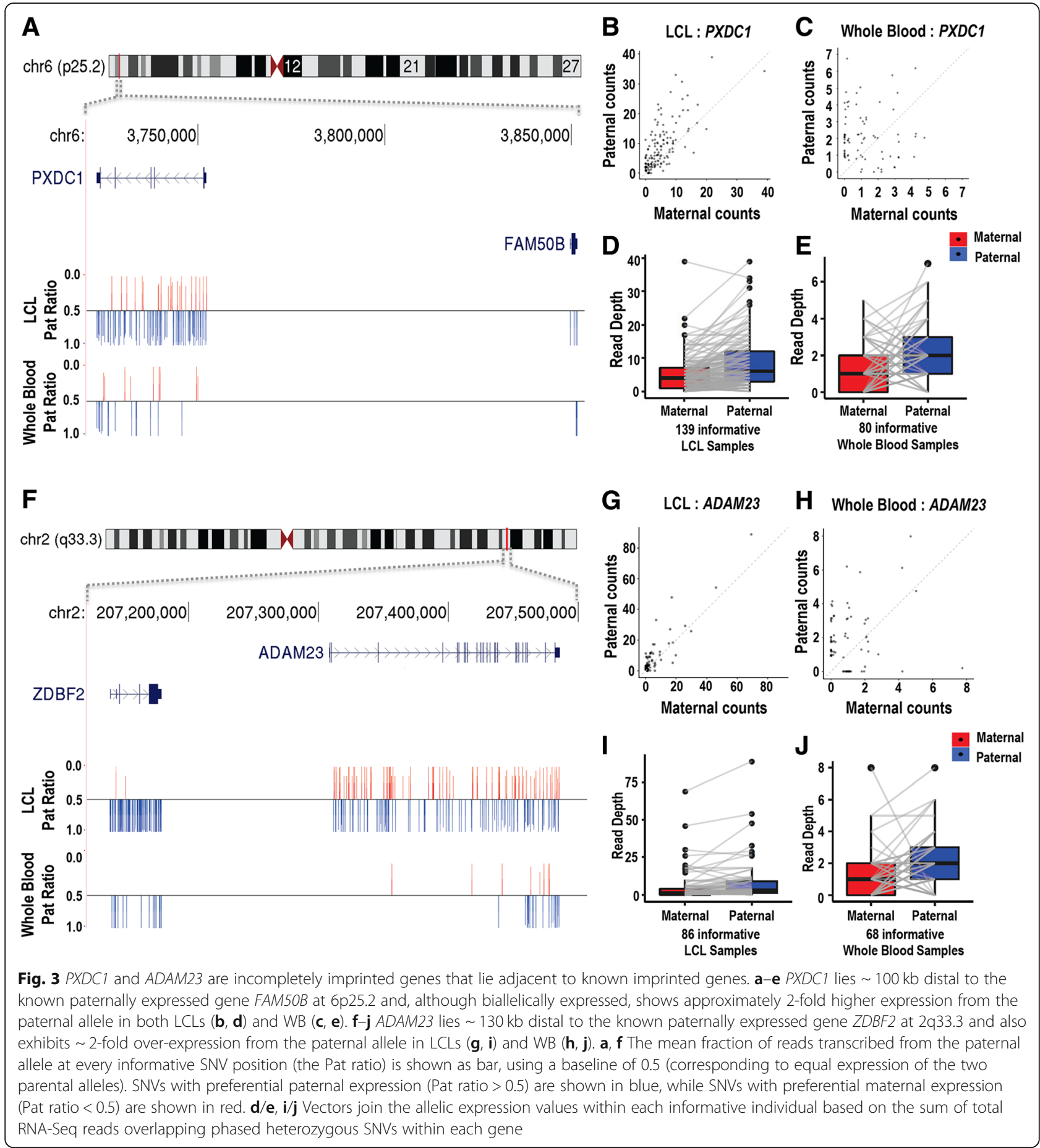

we choose a bin size of $25 \mathrm{~kb}$, as this is approximately midway between the median gene size $(\sim 30 \mathrm{~kb})$ and the median UGF size $(\sim 20 \mathrm{~kb})$. Within each bin, we aggregated maternal and paternal read counts for all available heterozygous SNVs and calculated the WSR $p$ value for parental expression bias for each $25 \mathrm{~kb}$ window. We took the set of all $25 \mathrm{~kb}$ non-overlapping windows that lie within $\pm 250 \mathrm{~kb}$ of strongly imprinted genes (those with paternal ratios $\leq 0.1$ or $\geq 0.9)$, removing any windows that overlapped other strongly imprinted genes, and compared the $p$ values for parental expression bias in the resulting set of $17525 \mathrm{~kb}$ windows versus all $25 \mathrm{~kb}$ windows in the rest of the genome $(n=58,951)$. We observed that regions surrounding the strongly imprinted genes are significantly enriched for signals of parental expression bias (permutation $p=0.0005$ ). Thus, 
our observations extend the known clustering of imprinted genes in the mammalian genome, showing that the effects of genomic imprinting can extend over broad regions and cause genes to show differing extents of parentally biased expression.

In another example, we identified two anonymous transcripts RP11-134O21.1 and GS1-57L11.1 at 8p23.2 as showing a $\sim 2: 1$ preferential expression of the paternal allele (Fig. 4). Consistent with our observations, RP11-134O21.1 has been previously reported as showing signs suggestive of imprinting [11]. Additionally, our previous studies of blood samples from patients with uniparental disomy (UPD) [25] identified a maternally methylated region located at the bidirectional promoter of these two transcripts, thus providing independent validation of our results.

\section{Strand-specific RNA-Seq data provides improved} resolution in cases of overlapping sense/antisense genes In LCLs, the availability of strand-specific RNA-Seq data allowed the quantification of maternal and paternal counts from the forward and reverse strands separately. In the majority of cases, the results obtained using stranded data were very similar to those obtained when aggregate data from both strands were considered. However, at the loci where overlapping genes were transcribed from both forward and reverse strands, the results gained using unstranded RNA-Seq sometimes yielded misleading results that differed from those obtained using stranded data. For KCNQ1/KCNQ1OT1，RB1/LPAR6, BMP8A/PPIEL-RP1169E11.4, and PER3/RP3-467L1.4, only the use of strandspecific data was able to unambiguously determine the correct imprinting status of these genes (Fig. 5). Consistent with prior studies of these loci, strand-specific data demonstrated that several sense and antisense transcript pairs displayed opposite parental bias: well-known examples of such scenario are KCNQ1 which is maternally expressed, whereas KCNQ1OT1 is paternally expressed [26]. Another example is $R B 1$, which is maternally expressed, whereas LPAR6 is paternally expressed (Fig. 5 and Table 1).

Imprinting patterns at the loci with multiple isoforms and overlapping transcripts

Previous studies have noted complex patterns of imprinting at certain genomic loci, such as isoform-specific

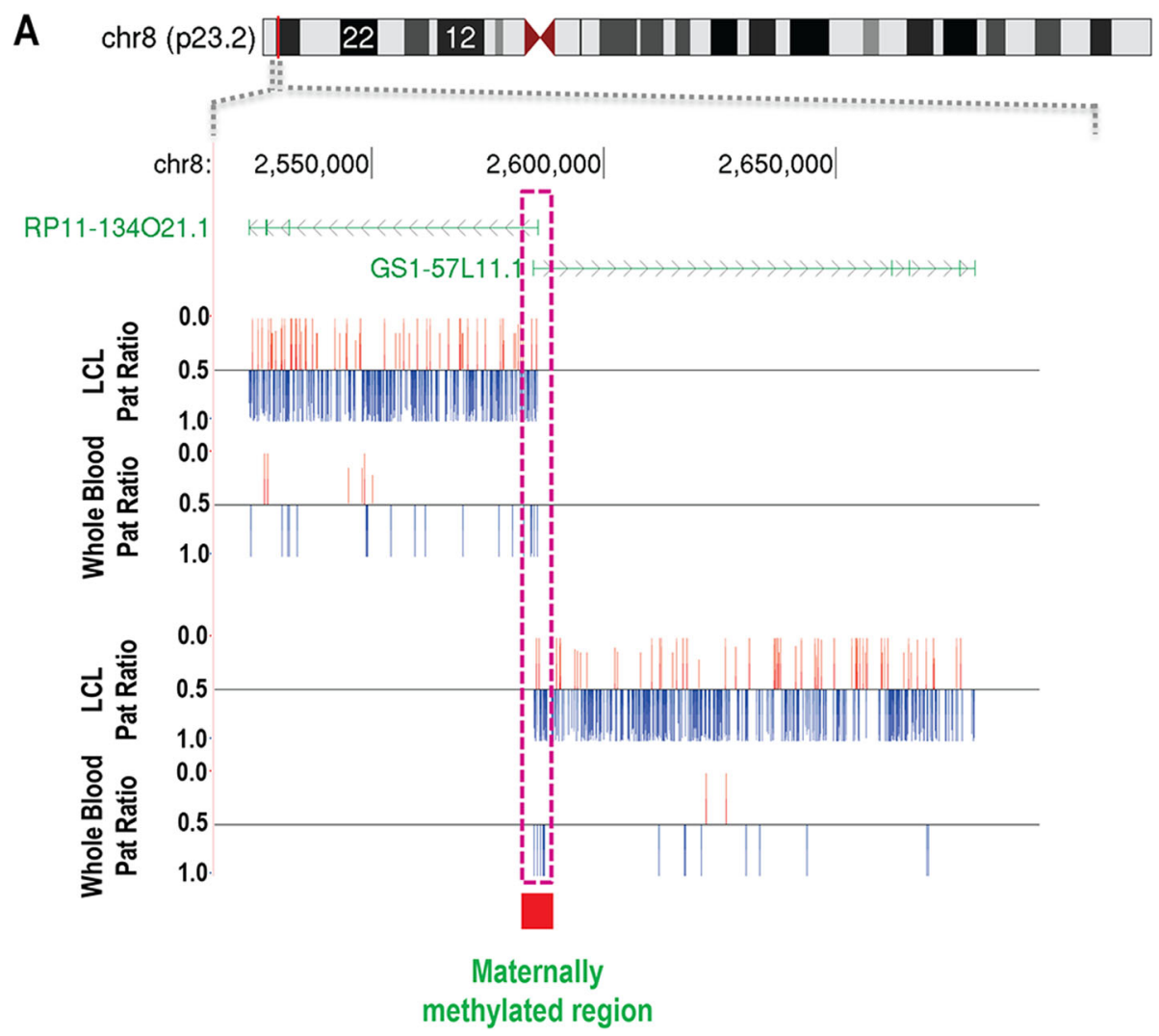

Fig. 4 Two imprinted transcripts located at 8p23.2 share a bidirectional promoter that coincides with a maternally methylated locus. RP11-134021.1 and GS1-57L11.1 are expressed from opposing strands, and both show $\sim 2$-fold expression from the paternal versus maternal allele in LCLs. Prior DNA methylation studies [25] identified a region of increased maternal methylation located at the shared promoter of these two transcripts, confirming parent-of-origin effects at this locus, and indicating this as the likely regulatory element controlling imprinted expression at this locus 


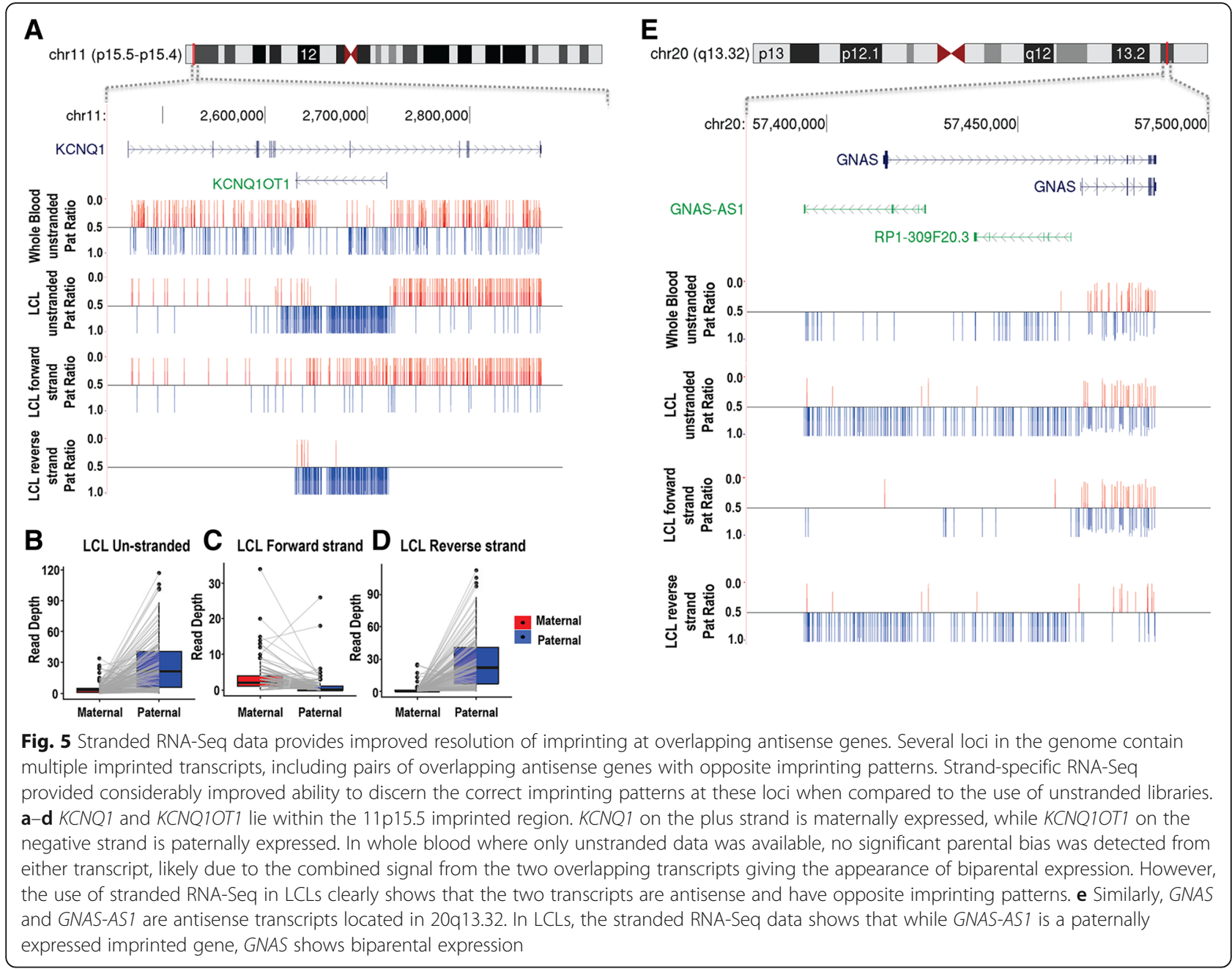

imprinting, or imprinted genes that overlap with other non-imprinted genes [24]. Using data from the location of individual informative SNVs within the imprinted genes we report, we identified several loci that exhibited differential imprinting patterns among subregions of gene annotations.

One example of this phenomenon is ZNF331, which has multiple different isoforms with different transcription start sites. As shown in Fig. 6, isoforms of ZNF331 that start at the most proximal promoter show no evidence of imprinting, while other isoforms transcribed from more distal promoters show 90\% expression from the paternal allele. Previous reports [24] have suggested that in the blood leukocytes, there is maternal-specific expression from the most proximal promoter of ZNF331, while our analysis indicates that in LCLs, these isoforms show equal biparental expression.

The data for HM13 suggest that this may show isoform-specific imprinting, with the longest isoforms showing a strong paternal expression bias, while shorter isoforms are biparentally expressed in the blood. An alternative possibility is that there is a parent-of-origin sensitive use of the most distal of the alternative polyadenylation sites in HM13 as a consequence of the imprinting of the MCTS2 gene, similar to what is observed in mice $[28,29]$ (Additional file 12).

Another example of similar complexity is the NAA60/ ZNF597 locus, which from prior studies is known to show isoform- and cell type-specific imprinting $[16,30]$. Additionally, this locus contains multiple overlapping transcripts, only some of which are imprinted. The longest isoform of NAA6O (forward strand) overlaps several other genes on the same or opposite strand, including ZNF174, ZSCAN32 LA16C-306E5.3, and MTRNR2L4. With strand-specific data and UGF annotations, we observed that the SNVs that overlap either ZSCAN32 (reverse strand), LA16c-306E5.3 (forward strand), or MTRNR2L4 (reverse strand) show no evidence of parental expression bias, while SNVs that fall uniquely within NAA60 or ZNF597 show almost exclusive maternal expression (Fig. 6). 


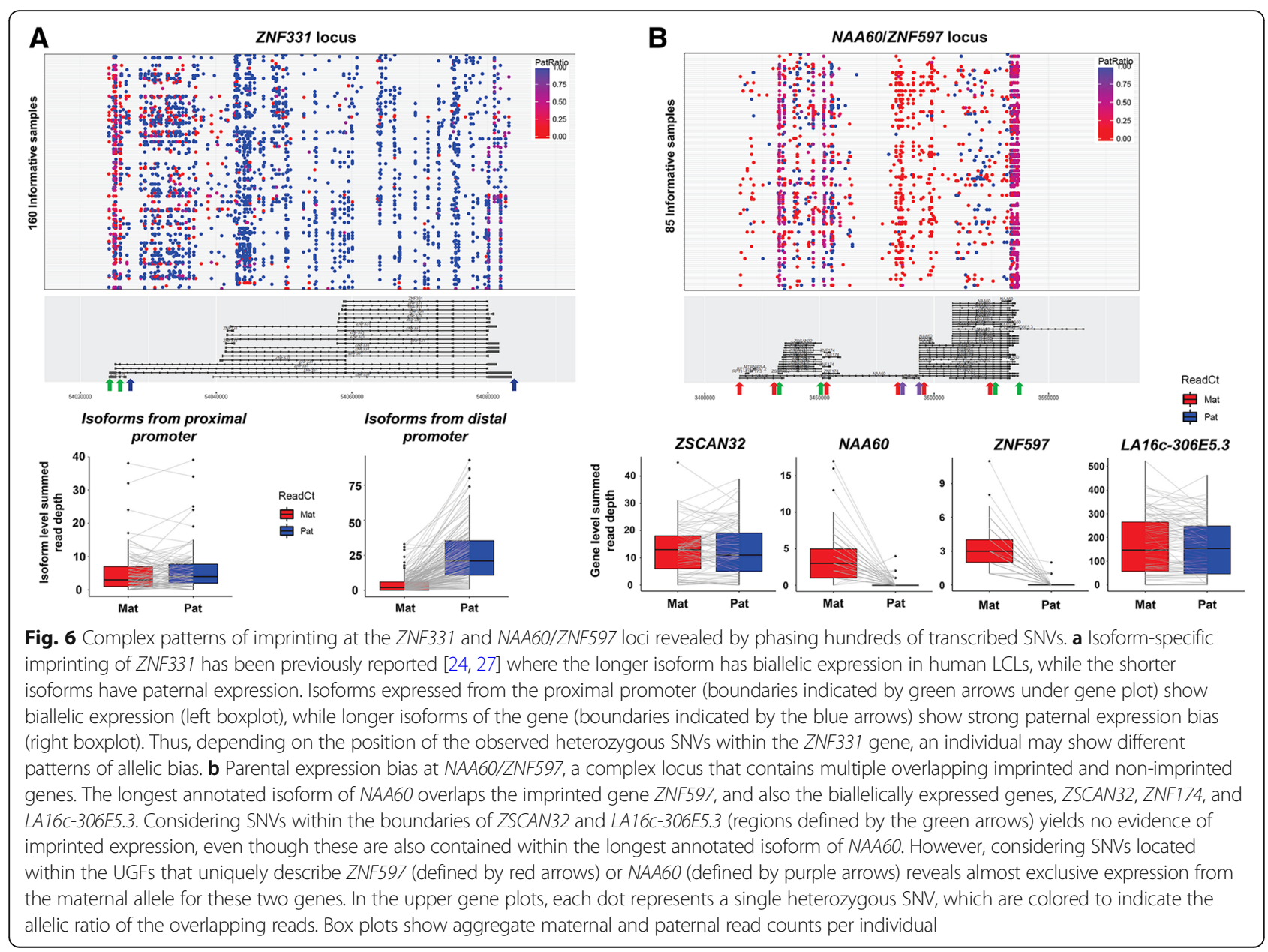

Finally, careful inspection of the TRAPPC9 locus enabled us to refine the signal of imprinting specifically to PEG13, which lies intronic within TRAPPC9. Here, we observed a cluster of SNVs located in the center of the annotated TRAPPC9 locus showing almost exclusive paternal expression, while SNVs located elsewhere in TRAPPC9 showed equal expression from the maternal and paternal alleles (Additional file 12). Although the gene annotations we used (Gencode v16) includes multiple isoforms of TRAPPC9, none included exons that corresponded to the cluster of paternally expressed SNVs within TRAPPC9. Instead, the use of Refseq gene annotations included the 5.6-kb transcript PEG13 (paternally expressed gene 13) that, like TRAPPC9, is expressed from the negative strand and coincides perfectly with this cluster of paternally expressed SNVs that lie intronic within TRAPPC9. Thus, careful curation of this locus revealed that the imprinted signal we observed in the blood comes solely from PEG13 and that the larger TRAPPC9 gene is not imprinted in the cell types we studied. Thus, our observations in LCLs and blood are consistent with previous studies made in the human brain [31].
Genome-wide scan for imprinting outside of known gene annotations

In order to search for novel signatures of imprinting outside of current gene annotations, we utilized a sliding window approach to systematically analyze the entire genome in an unbiased fashion. We chose a window size of $25 \mathrm{~kb}$ as this was close to the median transcript length, with a 5-kb incremental slide. At each position, we aggregated maternal and paternal read counts for all available heterozygous SNVs within the 25-kb window and calculated the WSR test statistics (Additional file 13) . Using this approach, as expected, we identified significant associations at nearly all imprinted genes found using our gene-centric approach. In several cases (e.g., ZNF331 and ZDBF2), significant signals of imprinted expression were observed downstream of annotated genes, which might represent transcriptional read-through beyond annotated 3' boundaries (Additional file 14). However, we also identified a significant signal of expression outside of known gene annotations on 13q21.1 in the LCL population. Here, a cluster of 35 informative SNVs spread over $\sim 8 \mathrm{~kb}$ showed a strong paternal bias, with $87 \%$ of reads supporting transcription from the 
paternal allele in 73 informative samples. We propose that this represents a maternally imprinted transcript transcribed from the forward strand that apparently shares a bidirectional promoter with LINC00434 (Fig. 7). In support of this, data from the ENCODE Project in cell line GM12878 indicates the presence of an anonymous transcript at this position that is consistent in size and strand with our observations. There was no significant expression from this locus detected in the whole blood. Interestingly, a previous study [23] of DNA methylation in oocytes reported that the bidirectional promoter of LINC00434 has a profile consistent with maternal-specific methylation. Additionally, Zink et al. reported TARDBPP2 within this locus as a putatively imprinted transcript with paternal expression bias [19].

\section{Discussion}

Here, we report a detailed survey of imprinted gene expression in two human tissues. We used a robust pipeline, incorporating the latest methods for allele-specific expression analysis, including rigorous removal of reads with potential mapping bias. The availability of phased genotype information from whole-genome sequencing of trios enables the assignment of expression levels from the two parental alleles at $>2.8$ million transcribed SNVs, providing a direct approach to assess imprinting genomewide and thereby allowing us to detect subtle imprinting effects, including genes with incomplete imprinting.

Further, we developed a robust statistical framework to account for population heterogeneity of imprinting. While many previous studies have called events at the level of individual samples and variants, we studied nearly 300 independent trios and employed two complementary statistical tests that considered aggregated read counts at the gene level across the whole population. The paired WSR is a non-parametric test that has the advantage of a low false-positive rate, but with reduced power at small sample size and low expression (Additional file 2). In contrast, SB uses the zero-inflated negative binomial distribution to fit the data, well-suited

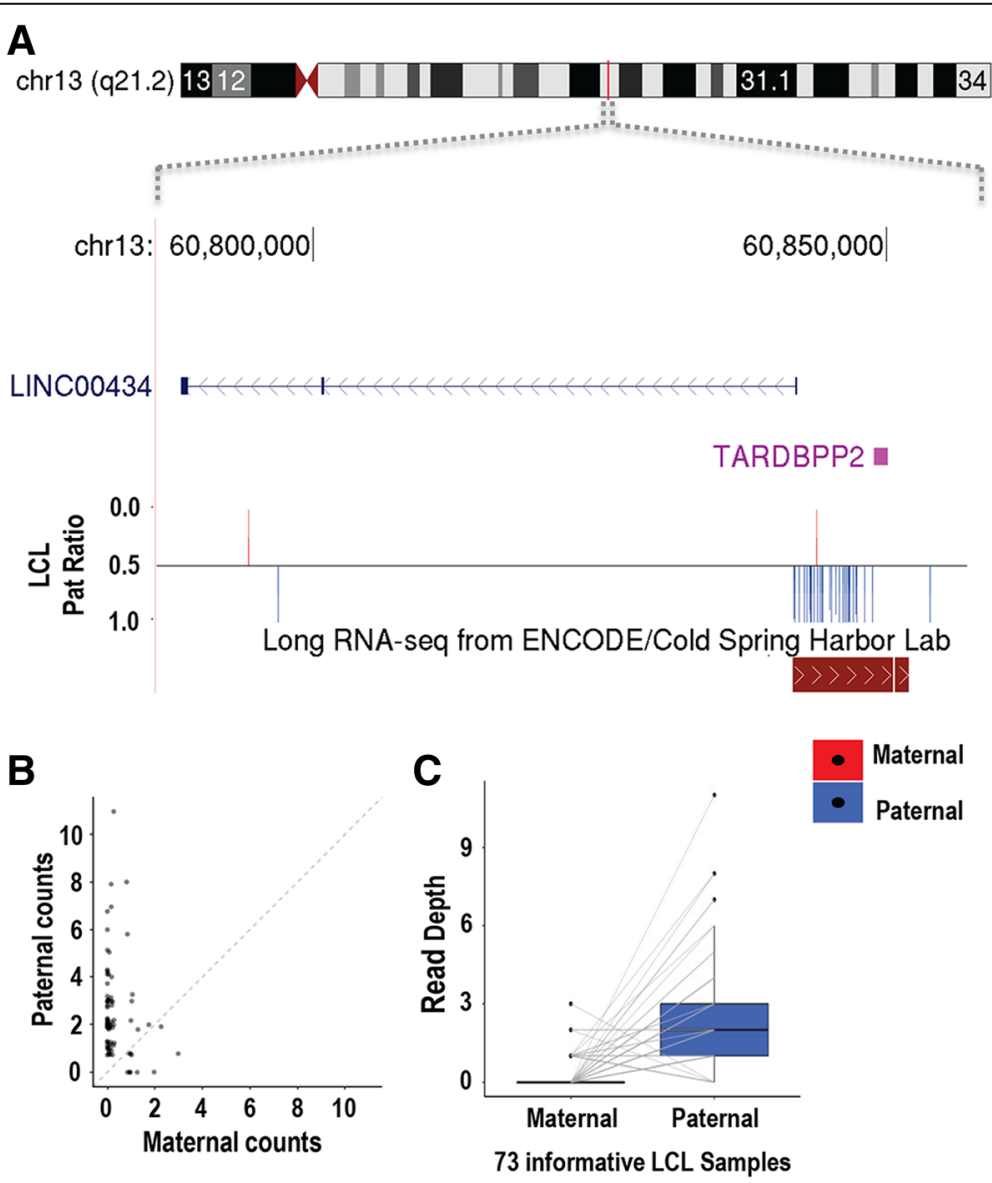

Fig. 7 a-c A putative imprinted IncRNA at 13q21.2. Using a sliding window analysis to interrogate the genome independent of gene annotations, we identified a cluster of 35 SNVs located in 13q21.2 (chr13:60,841,936-60,848,791, hg19) that showed a strong paternal expression bias. The putative transcript containing these SNVs is located on the forward strand and apparently shares a bidirectional promoter with the noncoding RNA LINC00434. This SNV cluster overlaps a putative anonymous transcript identified in LCLs by the ENCODE Project 
for zero-inflated count data such as RNA-Seq, providing increased power for genes with low expression. These approaches have the advantage of assessing the differences between paternal and maternal RNA-Seq counts at multiple heterozygous loci across all individuals simultaneously, thus providing both increased robustness and power to resolve subtle biases in expression from the two parental alleles, when compared to the study of single data points.

In a recent work by Zink et al., here, the authors utilized a different statistical analysis which uses a logistic regression framework to estimate PofO effect by modeling log odds ratio of reference and alternate read counts per SNV. The $p$ value of the top SNV, after multiple testing correction, is then assigned to the gene. A major difference with our study is the use of ref./alt ratios per SNV, instead of aggregated counts over all SNVs per UGF, as the basis for the statistical test. The number of informative samples and zero-inflation is important factors which are captured in our study by using two different tests, WSR and SB (Additional file 2). Aggregation of counts over multiple SNVs resolves to a certain extent the sparsity issue in our study, which may be negligible when sample size is large as is the case in Zink et al. In addition, genes with fewer SNVs will show a stronger PofO effect because of multiple testing correction at the gene level which is non-existent in our study due to aggregation.

Consistent with prior studies, we found that utilizing aggregated read counts across all heterozygous sites per gene in each individual, including intronic reads and SNVs covered by only a single read, gave the most power in our analysis [11, 15]. Finally, we filtered putative imprinted transcripts to remove false signals caused by reference bias, before manually curating each locus to resolve signals from overlapping and antisense transcripts. Importantly, curation to remove reference bias was an important step to avoid false-positive imprinting signals: despite the fact that we masked non-unique genomic regions and applied stringent filtering to remove reads with ambiguous mapping, we still identified several genes with significant signals of parental expression bias which were attributable to reads mapping preferentially to the reference sequence (as assessed by statistical comparison of coverage of the reference and alternative alleles) (Additional file 7).

Overall, this pipeline led to the identification of 45 imprinted genes and one imprinted unannotated transcript in 13q21.2. Of the imprinted genes identified, two notable examples are PER3 and IGF2BP3. PER3 [Period, Drosophila, homolog of 3; OMIM\# 603427] is a member of the Period family of genes and is expressed in a circadian pattern in multiple tissues [32]. PER3 is one of the several genes that regulate circadian rhythms and has been linked to seasonal affective disorder by both human and mouse studies [33, 34]. IGF2BP3 [insulin-like growth factor 2 mRNA-binding protein 3; OMIM\# 608259] binds to the 5' UTR of the imprinted gene IGF2, suggesting it has a role in the regulation of IGF2 production and is expressed ubiquitously across fetal and adult tissues [35, 36]. While previous reports have shown that IGF2BP3 is biallelically expressed, we identify a slight bias for increased expression from the paternal allele (LCL paternal ratio of 0.54). This may point at a coordinated PofO-based regulation of IGF2 signaling cascade. Notably, a maternally methylated $\mathrm{CpG}$ island associated with RPS2P32 gene lies $\sim 22 \mathrm{~kb}$ upstream of IGF2BP3 [25].

Classical studies of imprinting typically define imprinted genes as showing mono-allelic expression from just one of the two parental alleles. However, recent studies in mice have identified examples of incomplete, or non-canonical, imprinting [37]-such genes are biallelically expressed, but show a significant allelic bias, such that the two parental alleles are expressed at different levels. Our study also finds multiple examples of incomplete imprinting in the human genome, and we report nine imprinted genes that each shows consistent two- to threefold higher expression from the paternal allele. In several cases, these incompletely imprinted genes occur in close proximity to known imprinted genes that show mono-allelic expression, consistent with the known clustering of imprinted genes [38]. While it is possible that some of these genes with incomplete imprinting in the blood and/or LCLs might be fully imprinted (i.e., mono-allelically expressed) in other tissues, we note that none was found in a prior survey of imprinting that assayed 34 human tissues [11], making this unlikely.

Of note, we observed that some genes showed large apparent variations in paternal ratios (Fig. 2), and we found several different factors contributing to this phenomenon. In some cases, such as PXDC1 or PER3, this was apparently due to stochastic variation as a result of low read depth. For example, where an individual has a single heterozygous SNV in a gene that is covered by only two RNA-Seq reads, the possible paternal expression ratios are $0,0.5$, or 1 . Thus, in the case of a gene with low expression and incomplete imprinting, wide variations in the allelic ratios among different individuals will be observed as a result. In other cases, apparent variability of allelic ratios could be attributed to the fact that some genes showed isoform-specific imprinting patterns. For example, ZNF331 has multiple different isoforms with different transcription start sites: in LCLs, those transcribed from the distal promoters show 90\% expression from the paternal allele, while isoforms transcribed from the most proximal promoter showed no evidence of imprinting. Thus, depending on the position of heterozygous SNVs within ZNF331 carried by any 
one individual, the allelic ratio varied accordingly. Similar variability was also observed for $N A A 60$, stemming from the fact that there are several overlapping annotated genes at this locus, all of which have much higher expression levels in LCLs than NAA60. As a result, the paternal ratio of any one SNV within NAA60 is highly dependent upon its position within the locus. SNVs that overlap either ZSCAN32, ZNF174, or LA16c-306E5.3 showed no evidence of parental bias, while SNVs in regions that overlap only NAA60 or ZNF597 showed almost exclusive maternal expression (Fig. 6).

In addition to a gene-centric approach, we also utilized a sliding window analysis to screen for imprinted transcription across the genome, independent of known transcript annotations. This identified an imprinted locus at 13q21.2, apparently corresponding to an anonymous lncRNA approximately $8 \mathrm{~kb}$ in length. This imprinted transcript is antisense to LINC00434, with the two genes apparently sharing a bidirectional promoter. Although we did not detect any expression from LINC00434 in LCLs, given that these two genes are likely transcribed from the same promoter, we hypothesize that LINC00434 may also be imprinted. However, this hypothesis requires formal testing in other tissues to confirm if LINCO0434 is indeed imprinted.

Given a previous report of sex-specific variations in imprinting [11], we tested whether age or gender influenced the imprinting status for any of the 46 imprinted transcripts we identified. However, we did not detect any significant effects of these two variables on parental expression bias (Additional file 15). Furthermore, as studies in mice $[39,40]$ have previously identified a cluster of imprinted genes on the $\mathrm{X}$ chromosome, and phenotypic studies in humans have led to the suggestion that genes on the human $\mathrm{X}$ chromosome may also be subject to imprinting [41], we specifically searched for imprinting on the $\mathrm{X}$ chromosome. Although this analysis utilized only female samples, and thus suffered a reduction in power compared to our analysis of the autosomes, we were unable to detect any evidence to support the presence of imprinted genes on the human $\mathrm{X}$ chromosome.

In order to compare our results with those published in the literature, we performed a systematic survey of genes reported as imprinted in four other populationbased studies that have used RNA-Seq (Additional files 6 and 16). Overall, we observed moderate concordance among different studies, with 66 genes being reported by multiple studies and a further 159 genes reported in only a single study. Seven transcripts we identified as showing parental bias in gene expression were not reported in any of the other four studies (EHHADH, IGF2BP3, NEK10, PEG13, PRR25, RP11-64J4.2, ZNF613), while the majority of other singleton observations were made in the studies of Zink et al. and Babak et al. [12, 19]. While it is possible that some of these singleton observations might represent false positives, we suggest that the two major factors influencing whether a gene is reported as imprinted by a given study are likely the tissue or cell type studied, and statistical power of the study. As many genes show tissue-specific imprinting, any one study is therefore limited to observing only those genes that are imprinted in the cell type(s) being assayed. Power to discriminate significant parental expression bias is largely a function of sample size, with a large sample size allowing much more subtle PofO bias to be detected. However, power is also influenced by other factors such as the depth of RNA-Seq data obtained and the distribution of informative SNVs (which is related to both how the underlying genotypes were ascertained and sample ethnicity). These two factors largely explain why studies of multiple different tissues using GTEx data [11], and the recent study of $>11,000$ individuals from the Icelandic population [19], each detected many imprinted transcripts that were not observed in other studies. Other technical factors, such as gene annotations used and experimental (e.g., the use of unstranded versus stranded RNA-Seq, or polyA+ versus ribosome-depleted RNA) and statistical methodologies (e.g., whether data is analyzed at the level of individual SNVs, or aggregated across entire transcripts, and differences in the gene annotations used), likely account for the remaining differences among studies. Such factors make it difficult to directly compare results among studies. For example, of the five studies we compared, ours was the only one to report the known imprinted gene PEG13, which probably results from this transcript being absent in many gene annotation sets.

However, for ten genes that were reported as imprinted in the GTEx cohort, we did not observe evidence of imprinting, despite these genes having sufficient informative SNVs to be adequately assessed in our samples (UTS2, MEST, UBE3A, PLAGL1, CPA4, MAGI2, INPP5F_V2, PRSS50, THEGL, RP11-7F17.7). We note that of these ten genes, MEST, UBE3A, PLAGL1, CPA4, $M A G I 2$, and INPP5F_V2 have all been reported as imprinted in other prior studies. While it is possible these may represent false negatives in our analysis, many apparently show tissue-specific imprinting, with normal biparental expression in the blood and LCLs, thus explaining our results [42-45]. In addition, we note that UTS2 overlaps and is antisense to PER3, a gene which we identify as showing a weak paternal bias in LCLs. Given our improved methodology that utilized strandspecific RNA-Seq, we suggest that the previously reported imprinting of UTS2 instead likely reflects paternally biased expression of PER3. Given the improved resolution of strand-specific over unstranded RNA-Seq 
data, we suggest that future expression-based studies of imprinting should utilize this approach where possible.

Our study has some limitations. Primarily, as our approach relies on measuring read depth over transcribed SNVs, we were limited to the study of genes that both contained heterozygous variants and were expressed at sufficient levels to be analyzed. Thus, genes that were not expressed at detectable levels in a sufficient number of individuals, or which lacked heterozygous variants in our samples, were not assayed. Similarly, we had a little discriminatory power to detect imprinting for genes that contained very few SNVs in our cohort or for those that were expressed at very low levels. Further, as we studied samples of peripheral blood and LCLs, we were unable to detect genes that show imprinting confined to other tissues [11]. Finally, as the LCLs we studied are immortalized cell lines, it is possible this process may have disrupted epigenetic processes such as imprinting. However, arguing against this possibility, there was both strong concordance of our results obtained in LCLs with previous studies of imprinting, and several of the imprinted genes detected in LCLs were also supported by methylation and/or RNA-Seq data from the whole blood [11].

We are aware that some previous studies have suggested that independent validation such as pyrosequencing is necessary for the robust identification of imprinted loci from RNA-Seq data [15]. This is particularly true where statistical models assume random independent sampling of reads and do not account for technical and biological variation. DeVeale et al. also suggested, based on pyrosequencing validation, several characteristic features tend to associate with genuine imprinted genes, the most prominent one of which is the presence of concordant signals of imprinting among neighboring SNVs in the same gene. In essence, this is exactly what our statistical approach does, as we chose an approach that aggregates data from multiple SNVs within each transcript annotation, thereby avoiding single SNV calls as a major source of false positives. A second feature associated with true positives highlighted by DeVeale et al. is the recurrence of a signal across biological replicates. Again, by determining the statistical significance for parental expression bias at the population level considering signal from all informative individuals, we automatically ensure recurrence across multiple individuals. In contrast, most pyrosequencing assays only assess allelic bias based on a single SNV.

\section{Conclusion}

Given that our study assessed the imprinting status of $41 \%$ of human transcripts, and identified 45 that are imprinted, our findings are broadly consistent with previous projections that have suggested that the human genome likely contains approximately 100 genes that are imprinted in somatic tissues [46].

\section{Methods}

Strand-specific RNA-Seq in $\mathbf{1 6 5}$ lymphoblastoid cell lines We generated RNA-Seq data from lymphoblastoid cell lines (LCLs) for 57 CEPH (CEU), 58 Yoruba (YRI), and 50 Han Chinese (CHS) samples, all of whom were offspring of multi-generation pedigrees studied as part of The HapMap (http://hapmap.ncbi.nlm.nih.gov/) and/or 1000 Genomes (http://www.internationalgenome.org/) Projects. Samples are listed in Additional file 17.

\section{Genotype data processing}

For 163 samples, genotype data from the complete mother/father/child trio were available, while for the two samples, genotype data for only one parent was available. We obtained 1000 Genomes and HapMap Project data from multiple releases: this included data from The 1000 Genomes Project phase 1 and phase 3 generated from low-coverage Illumina whole-genome sequencing, high coverage Complete Genomics whole-genome sequencing data, exome sequencing, Illumina Omni 2.5M SNV array data, and HapMap3 Project data genotyped on Illumina $1.6 \mathrm{M}$ and Affymetrix 6.0 SNV arrays. We included highquality filtered and curated DNA genotype data from the final releases of all these resources and combined into population-specific datasets. We performed quality control on the merged data such as resolving strand inconsistencies, removing multi-allelic SNVs and indels, removing SNVs not present in the 1000 Genomes data, and converting coordinates from hg18 to hg19 where required using PLINK (versions 1.07 and 1.9) [47, 48], vcftools (version 0.1.15) [49], and Beagle Utilities.

Due to the differing genotyping approaches and resulting SNV densities available across different individuals, we performed combined imputation and phasing to increase SNV density and infer the two parental haplotypes in each offspring with Beagle 4.0 [50]. This used family pedigree information with the 1000 Genomes phase 3 reference panel downloaded from the Beagle website (http://bochet.gcc.biostat.washington.edu/beagle/1000_Genomes_phase3_v5a/). Using 493 HapMap samples from the CEU, YRI, and CHS populations, we created population-specific reference panels to improve the imputation accuracy. Since many of the samples in our target panel are also part of 1000 Genomes Project reference panel, for each population group, we created subsets of target and reference panel in such a way that there are no overlapping samples in two sets and imputed and phased each of these subsets of target panel separately. Each chromosome was divided into segments to efficiently perform imputation and phasing, and these segments were subsequently merged together to yield 
chromosome-wide imputed and phased genotypes. Imputed genotypes were filtered to retain only high-quality genotypes $\left(R^{2} \geq 0.95\right)$. We also removed sites with Mendelian errors in each trio, Hardy-Weinberg equilibrium $p<10^{-4}$, and retained only biallelic SNVs with minor allele frequency $\geq 5 \%$ in at least one of the three ethnicities in the cohort. This yielded $\sim 3.9$ million high-quality SNVs phased for parental origin.

To reduce phase switch errors introduced during phasing that would result in incorrect parental origin assignment of SNVs, we used an R script developed in-house (https://github.com/SharpLabMSSM/PofOAssignment).

This method utilizes the phased genotypes generated using BEAGLE, as follows: Each offspring's haplotype is compared with the parental haplotypes using a sliding window of $100 \mathrm{SNVs}$ with $50 \mathrm{SNV}$ incremental slide. Within each window, we check for perfect matches between each offspring haplotype and the four possible haplotypes within the parents. Parental origin assignments for each haplotype in the offspring are based on an unambiguous match to a single parental haplotype. This approach allows assignment of parental origin at uninformative sites where all members of the trio are heterozygous and also provides an error check for phase switching. In the case when offspring's haplotypes do not perfectly match a parental haplotype, the genotypes in the window are set to missing. Subsequently, we then recover any such lost sites using simple rules of Mendelian inheritance to each individual SNV genotype in the trio. Thus, by using a combined approach leveraging both statistical phasing with rules of Mendelian inheritance, we are able to generate maximally informative assignment for parental origin at heterozygous SNVs, with a minimal error rate.

\section{Sample preparation}

Lymphoblastoid cell lines were obtained from the Coriell Institute (Camden, NJ). Cells were grown in RPMI1640 media supplemented with $1 \mathrm{mML}$-glutamine, $10 \% \mathrm{FBS}$, and $100 \mathrm{u} / \mathrm{L}$ each of penicillin and streptomycin, according to the recommended protocols. Total RNA was extracted from frozen cell pellets (5-10 million cells) using TRIZOL, according to the manufacturer's instructions (ThermoFisher Scientific). Strand-specific RNA-Seq libraries were prepared using NEBNext Ultra Directional RNA Library Prep Kit from Illumina. One microgram of total RNA was used as input, polyA+ selected, followed by strand synthesis was performed. Libraries were sequenced on an Illumina Hiseq 2500 instrument, with 10 samples pooled per lane, to generate $100 \mathrm{bp}$ single-end reads to a median depth of $\sim 16$ million reads per sample.

\section{RNA-Seq data processing}

Quality control analysis was performed on RNA-Seq reads using fastqc (version 0.11.2) (http://www. bioinformatics.babraham.ac.uk/projects/fastqc). Overrepresented sequences were removed using trimmomatic (version 0.32) [51], and trimmed reads $\geq 30 \mathrm{bp}$ in length were kept. Cleaned reads were mapped to the human reference genome (hg19) with Gencode v16 annotations using the STAR aligner (version 2.3.0) [52], yielding a mean of $79 \%$ uniquely mapped reads. Picard (version 1.112) (https://github.com/ broadinstitute/picard) was used for intermediate BAM file processing such as add read groups and sorting and merging BAM files of the same samples. To correct for mapping errors and biases which can result in false-positive allele-specific read assignments, we used a collection of utilities in the WASP software (version 0.1) [53], resulting in the removal of a mean of $36 \%$ of reads that overlapped SNVs in each sample, for which unambiguous allelic assignment could not be made. After parental origin assignment for SNVs in each offspring, heterozygous sites were used to determine allele-specific expression. We first quantified reference and alternate RNA-Seq reads mapped at heterozygous loci using AlleleCounter (v0.2, https://github.com/secastel/allelecounter) implemented in Python [10]. Then, reference and alternate allele counts were used with PofO information to assign counts to the maternal and paternal alleles at each heterozygous site. Reads that did not uniquely map, or had base quality $\leq 10$, were discarded. To further reduce the mapping errors, we applied additional filters, removing heterozygous SNVs that (i) had a mappability score $<1$ (based on the "CRG GEM Alignability of 50mers with no more than 2 mismatches" track, downloaded from UCSC genome browser), (ii) overlapped CNVs with $\mathrm{MAF} \geq 5 \%$ identified in samples from the 1000 Genomes and HapMap Projects (ftp://ftp.1000genomes.ebi.ac.uk/vol1/withdrawn/phase3/integrated_ sv_map/ and common CNVs [54], (iii) segmental duplications, and (iv) simple repeats (both downloaded from "Variation and Repeats" track group of the UCSC genome browser). These filters resulted in the removal of $21 \%$ of heterozygous sites, leaving 3.1 million sites for downstream analysis.

\section{Unstranded RNA-Seq in 131 whole blood samples}

The Genome of the Netherlands (GoNL) Project [20] performed whole-genome sequencing of 250 family trios, a subset of which also had whole blood transcriptomes sequenced as part of the BBMRI-NL Biobank-based Integrative Omics Study (BIOS) $[55,56]$. From these, we utilized data from 131 children with whole blood RNASeq data that passed all quality criteria and had genotypes concordant with those obtained by whole-genome sequencing (listed in Additional file 18). The individuals 
were participants from one of four biobanks: LifeLinesDEEP, The Leiden Longevity Study, Netherlands Twin Registry, and the Rotterdam Study.

\section{Genotype data processing}

DNA genotypes of 250 Dutch families were phased and imputed using BEAGLE [57] and IMPUTE2. An integrated phase panel was constructed using SNV genotype likelihoods from the GATK:UnifiedGenotyper as input for BEAGLE, treating all samples as unrelated. SHAPEIT2 and MVNcall19 were then used along with trio information to phase the complete set of SNVs. Each haplotype transmitted to the offspring, and therefore, allelic parental origin was then obtained from the phased haplotypes [20].

\section{Sample preparation}

Total RNA from the whole blood was treated using Ambion's GLOBIN clear kit and subsequently processed for sequencing using the Illumina Truseq version 2 library preparation kit. Paired-end 50 bp reads were generated using an Illumina HiSeq 2000 instrument, pooling 10 samples per lane. Read sets per sample were generated using CASAVA, retaining only reads passing Illumina's chastity filter for further processing. Data was generated by the Human Genotyping Facility (HugeF) of ErasmusMC (The Netherlands, see URLs). Full details are described in [55].

\section{RNA-Seq data processing}

Initial quality control was performed using FastQC (v0.10.1). Removal of adaptors was performed using Cutadapt (v1.1) [58]. Sickle (v1.2) [59] was used to trim low-quality ends of the reads (minimum length 25 , minimum quality 20). The reads were mapped with the STAR aligner (v2.3.125) [52] to human reference genome hg19 masked at all single nucleotide variants with MAF $>0.01$ in GoNL samples. Full details are described in [55]. To reduce the influence of reference bias, we utilized WASP (version 0.1) [53] to remove reads that aligned to different genomic positions after substituting the variant site. A summary of the influence of masking SNV positions in the reference and utilizing WASP to remove reads that show ambiguous mapping positions is shown in Additional file 19.

To obtain the parent-of-origin allelic counts, we first computed RNA-Seq reference and alternative counts using the GATK (v3.6-0-g89b7209) ASEReadCounter tool [60]. A script was then used to re-label the reference and alternative counts with parental origin based on the transmitted allele, leaving $\sim 0.9$ million heterozygous sites with paternal and maternal read counts for downstream analysis. A summary of the complete analytical pipeline is shown in Additional file 20.

\section{Statistical analysis to identify imprinted expression}

Since overlapping genes are common in the eukaryotic genome [61], care must be taken when assigning reads to specific transcripts. To avoid misassignment of reads at SNVs located within the overlapping transcripts, we compiled all genes from Gencode annotations into a model where we consider the overlapping regions of different genes as a separate unit, termed as "unique gene fragments" (UGFs) (Additional file 21). The resulting gene models comprised 79,452 UGFs and were used for assigning each heterozygous SNV to specific genes.

To maximize the statistical power for detecting PofObiased expression, we summed the read counts for all SNVs within each UGF. We calculated the paternal allelic ratio (defined as the fraction of reads derived from the paternally inherited allele) for each individual using aggregated read counts across all informative SNVs within each UGF. We used the paternal allelic ratio of each informative individual to calculate the mean paternal ratio per UGF.

To formally test for parental bias in the expression of UGFs, we utilized two complementary statistical approaches. We chose (i) a frequentist non-parametric approach, the paired Wilcoxon signed-rank (WSR) test and (ii) an empirical Bayes approach ShrinkBayes [62]. ShrinkBayes computes a Bayesian false discovery rate (BFDR), and we applied Benjamini-Hochberg false discovery rate (FDR) correction to the results of the WSR test, considering those UGFs with FDR $q<0.1 \quad(10 \%$ FDR) as showing significant evidence of imprinting. In each cohort, we only considered results for those genes in which at least $10 \%$ of individuals had $\geq 1$ read informative for parental origin. Based on the results of these two tests, we classified predicted imprinted genes into those with high confidence (identified as significant by both tests) and low confidence (significant by one of the two tests). WSR test is a paired difference non-parametric test. It assigns ranks to the paternal/ maternal differences with $H_{0}$ : mean difference in pairs is symmetric around 0 . The test is robust against outliers and has no distributional assumption. ShrinkBayes is an advanced statistical method specifically designed to handle zero-inflated count data allowing multiparameter inference and modeling of random effects in a Bayesian setting. It relies on INLA [63] for the parameter estimation per gene while borrowing information across genes by empirical Bayes-type shrinkage of parameters. It allows a spike-and-slab prior for the parameter of interest (patmat: mean difference in pairs) to test $H_{0}$. Per UGF, we use a simple model with a single predictor parameter for imprinting (patmat) and a random effect parameter (indiv) to account for within-individual variability. 


$$
y \sim 1+\text { patmat }+f(\text { indiv })
$$

To assess the performance of the test procedures ShrinkBayes and WSR, we developed a simulation scheme. ShrinkBayes is superior to WSR in terms of statistical power (Additional files 1 and 2) at a cost of increased computational resources. Using the two tests together reduces the false-positive rate (Additional file 1), which motivates our definition of high-confidence genes.

Following statistical testing, we manually curated the UGF level results based on visual inspection of data plots, considering both gene annotations and strandspecific data in LCLs. Here, we removed redundancies, and in the case of overlapping transcripts, assigned imprinted expression to the correct gene. At several loci where we detected imprinted expression, gene annotations included transcripts with anonymous clone IDs. An example of this is the L3MBTL1/SGK2 locus on chromosome 20. Here, Gencode annotations include a transcript RP1-138B7.5, which is almost identical to an isoform of $S G K 2$. In such cases, even though the transcript RP1-138B7.5 was included in our initial list of significant imprinted genes, to avoid artificially inflating the number of imprinted transcripts we report, where these anonymous clone IDs likely corresponded to other annotated genes, we did not report them in our final curated list (Tables 1 and 2). Furthermore, although we filtered reads for potential mapping bias using WASP, we performed an additional check of UGF-level data for reference bias. We aggregated reference and alternate allele read counts at the UGF level and applied a twosided WSR test to check whether the distribution of reference and alternate read counts was significantly different after multiple testing corrections (5\% FDR), removing genes that showed significant reference bias.

\section{Chromosome $X$ analysis}

To assess if any genes on the $\mathrm{X}$ chromosome were expressed in a PofO-specific manner, we conducted analyses of female samples in both LCLs $(n=68)$ and WB $(n=77)$ samples, taking into account the potential confounder of unequal $\mathrm{X}$ chromosome inactivation ratios (XCIR). In each female, we used maternal and paternal read counts data for all X-linked genes containing heterozygous variants to calculate the XCIR:

$$
\mathrm{XCIR}=\frac{\sum_{i=1}^{m} \text { patCount }_{i}}{\sum_{i=1}^{m} \text { patCount }_{i}+\sum_{i=1}^{m} \text { matCount }_{i}}
$$

where $m$ is the number of genes. We excluded females with skewed XCIR (ratios either $<0.2$ or $>0.8$ ), which left 45 females in the LCL and 67 in WB cohort. In the remaining females, we adjusted the maternal and paternal read counts of X-linked genes using the XCIR measured in each individual. Finally, we applied the paired
Wilcoxon signed-rank test using the XCIR-weighted maternal and paternal read counts of X-linked genes.

\section{Additional files}

Additional file 1: Power estimates for ShrinkBayes and the paired Wilcoxon signed-rank test on the number of genes ( $\mathrm{L}$ ) and samples (R). To assess the performance of the test procedures SB and WSR test, we developed a simulation scheme with the number of genes and individuals as parameters. RNA Seq data is simulated using ssizeRNA R package (v1.2.8) capable of simulating count data for two-group differential gene expression analysis with additional parameters for fold change, dispersion, and size (expression level). We model imprinting in an individual with expression fold change in one of the parents. We labeled the groups as paternal and maternal and assigned a factor of 2 fold change to one group and thus simulating imprinting. For a better approximation of the real data, we generated different expression levels from low to high with different proportions and fixed dispersion to 0.4 . We use count level categories $\{2,10,20,50,100,500\}$ with corresponding proportions of genes $\{0.5,0.2,0.1,0.1,0.07,0.03\}$ having those count levels. Note that for the sake of approximation, we used fixed values 138 and 24,597 for the number of individuals and genes, respectively,

corresponding roughly to the reported aggregated GoNL data in the manuscript. The expression levels, dispersion, and fold change are fixed for all simulations. We also fix the number of imprinted genes to $1 \%$ of the total number of genes. The imprinting is simulated by assigning a factor of twofold change to the paternal label. The same expression level and proportions are used for the $99 \%$ non-imprinted gene but with fold change $=1$. (TIF $879 \mathrm{~kb})$

Additional file 2: Putative imprinted UGFs identified by ShrinkBayes and/or the paired Wilcoxon signed-rank test as a function of underlying sample size (L) and mean expression (R). Each box plot shows transcript fragments with significant evidence of imprinting that were (left) high confidence (identified by both SB and WSR tests), (middle) identified by SB only, and (right) identified by WSR only (FDR $q<0.1$ ). Each UGF was subject to manual curation of raw data and classified as a true positive (TP, blue) or false positive (FP, red). The LC category (SB and WSR) shows a clear difference in the test performance: SB is more sensitive at reduced sample size and expression, although WSR still identified many signals that are missed by SB. We conclude that signals of imprinting identified by both tests are the most robust, while each test is able to detect additional signals, albeit with a higher false-positive rate. (TIF $711 \mathrm{~kb}$ )

Additional file 3: 78 significant unique gene fragments. All UGFs with FDR $q<0.1$, prior to manual curation. (XLSX $113 \mathrm{~kb}$ )

Additional file 4: All unique gene fragments tested in this analysis. Data for all UGFs in the genome. (XLSX $19820 \mathrm{~kb}$ )

Additional file 5: Overlap of identified genes in two tissues and two statistical methods. (A) $51 \%$ of genes identified as imprinted genes were concordant in both LCLs and whole blood. (B) In LCLs, $89 \%$ of the genes that were scored as imprinted were detected by both Wilcoxon signedprank test and ShrinkBayes. (C) In the whole blood, $63 \%$ of the genes that were scored as imprinted were detected by both Wilcoxon signed-rank test and ShrinkBayes. (TIF $423 \mathrm{~kb}$ )

Additional file 6: Comparison of LCL/WB imprinting results with previous studies. We list all genes identified as imprinted either in our dataset, as well as those reported as imprinted in recent studies that used RNAseq data in either the GTEx cohort or the Icelandic population. (XLSX $41 \mathrm{~kb})$

Additional file 7: Reference bias can cause false-positive signals of imprinting. A screen for imprinted genes on the $X$ chromosome identified two putative imprinted transcripts, which were both found to be falsepositive associations due to reference bias. (A) UCSC Genome Browser view showing a single informative SNV within RNA28S5, a pseudogene at Xq22.3. (B) Scatter plot and (C) table of reference and alternate read counts in six female LCLs heterozygous for rs 190908473 shows that > 98\% of reads overlapping this SNP match the reference genome, indicating the putative maternal expression bias is caused by a read 
mapping bias. (D) ARSD showed a putative maternal expression bias in samples of whole blood. (E) However, informative RNA Seq reads from ARSD showed a strong mapping bias to the alternative (non-reference) allele, indicating this as a false-positive association. (TIF $588 \mathrm{~kb}$ )

Additional file 8: Results of clonality analysis in LCLs. Reanalysis of unique gene fragments with FDR $a<0.1$ using only 45 non-clonal female LCLs without skewed X chromosome inactivation ratios. (XLSX $20 \mathrm{~kb}$ )

Additional file 9: Methylation profiles at imprinted loci in whole blood, LCLs, and samples with genome-wide maternal or paternal uniparental disomy (matUPD and patUPD). To assess whether there is a loss of methylation at imprinted loci in LCLs, we gathered available Illumina 450 $\mathrm{k}$ methylation data from whole blood (1419 samples taken from six published studies on GEO) [67], HapMap LCLs (133 samples from GEO dataset GSE39672), and whole blood from individuals with genome-wide uniparental disomy (UPD) (taken from GEO dataset GSE52576). The plot shows mean DNA methylation levels at 48 differentially methylated regions associated with imprinted genes that show parental-specific methylation [24]. LCLs and whole blood show very similar methylation profiles at all imprinted DMRs. In contrast for the six paternally methylated DMRs (left side), methylation in maternal UPD samples is much lower than either blood or LCLs. Similarly, for the 42 maternally methylated DMRs (right side), samples with paternal UPD show much lower methylation than either blood or LCLs. Thus, we conclude that there is no evidence for loss of imprinting in LCLs and that methylation at imprinted DMRs is generally very similar in the blood and LCLs. (TIF $794 \mathrm{~kb}$ )

Additional file 10: Location of closest imprinted differentially methylated region from two published studies to each imprinted gene identified in our dataset. (XLSX $17 \mathrm{~kb}$ )

Additional file 11: Example of strand-specific data showing paternal expression bias at PER3/RP3-467L1.4 locus. PER3 and RP3-467L1.4 are two overlapping genes transcribed from opposite strands. PER3 shows incomplete imprinting, whereas RP3-467L1 shows stronger paternal bias in $L C L s$ (paternal ratios $=0.61$ and 0.81 , respectively). (TIF $2840 \mathrm{~kb}$ )

Additional file 12: Complex imprinting at HM13 and the TRAPPC9PEG13 locus. (A) The longest isoform of HM13 shows paternal expression bias, while shorter isoforms are apparently biallelically expressed. (B) PEG13 shows exclusive paternal expression, while TRAPCC9 is biallelic. (TIF $1281 \mathrm{~kb}$ )

Additional file 13: All $25 \mathrm{~kb}$ windows with significant $p$ values from sliding window analysis. (XLSX $478 \mathrm{~kb}$ )

Additional file 14: Examples of significant signals of imprinting that extend beyond the annotated boundaries of genes. Possible transcriptional read-through beyond gene annotations at ZNF331, ZDBF2, and GNAS-AS1 locus. (TIF $807 \mathrm{~kb}$ )

Additional file 15: Analysis of potential age and gender effects on imprinting. (PDF $111 \mathrm{~kb}$ )

Additional file 16: Venn diagram showing an overlap of imprinted genes reported by five recent studies that utilized RNA-Seq. (TIF $657 \mathrm{~kb}$ )

Additional file 17: $165 \mathrm{LCLs}$ used for RNA-Seq analysis and their parents. (XLSX $20 \mathrm{~kb})$

Additional file 18: 131 whole blood samples used for RNA-Seq analysis. (XLSX $12 \mathrm{~kb}$ )

Additional file 19: The effect of masking SNV positions and utilizing WASP on reference genome mapping bias. Utilizing an unmasked reference genome, the median alternate ratio was 0.458 . This increased to 0.483 after masking common SNV positions (SNVs with MAF $>0.01$ were replaced by " $\mathrm{N}$ ") and further increased to the theoretical expectation of 0.5 after utilizing WASP to remove reads with ambiguous mapping positions. (TIF $312 \mathrm{~kb}$ )

Additional file 20: A summary of the analytical pipeline used for identifying parental bias in gene expression in whole blood samples. (TIF $408 \mathrm{~kb}$ )

Additional file 21: Definition of unique gene fragments (UGFs). In order to avoid misassignment of reads at SNVs located within overlapping transcripts during our gene-centric analysis, we compiled all genes from Gencode annotations into a gene model where we consider overlapping regions of different genes as a separate gene. We termed these annotations unique gene fragments (UGFs). Statistical testing on each UGF was performed, and after all significant associations were compiled (Additional files 3 and 4), we manually curated each signal and removed redundant annotations, reporting a final list of 45 imprinted genes (Tables 1 and 2). (TIF $210 \mathrm{~kb}$ )

Additional file 22: Members of the Biobank-based Integrative Omics Study (BIOS) Consortium. (PDF $68 \mathrm{~kb}$ )

Additional file 23: Members of the Genome of the Netherlands (GoNL) Consortium. (PDF $43 \mathrm{~kb}$ )

\section{Abbreviations}

GoNL: Genome of the Netherlands; LCL: Lymphoblastoid cell line; PofO: Parent-of-origin; SB: ShrinkBayes; UGF: Unique gene fragments; WB: Whole blood; WSR: Paired Wilcoxon signed-rank test

\section{Acknowledgements}

We would like to thank Tuuli Lappalainen and Stephane Castel for facilitating the collaboration underlying this study. The research reported in this paper was supported by the Office of Research Infrastructure of the National Institutes of Health under award number S100D018522. The content is solely the responsibility of the authors and does not necessarily represent the official views of the National Institutes of Health. This work was supported in part through the computational resources and staff expertise provided by Scientific Computing at the Icahn School of Medicine at Mount Sinai. Work on the WB samples was performed within the framework of the Biobankbased Integrative Omics Studies (BIOS) Consortium and the GoNL Project which are funded by BBMRI-NL, a research infrastructure financed by The Netherlands Organization for Scientific Research (NWO project 184.021.007). The members of the BIOS and GoNL Consortia are listed in Additional files 22 and 23 , respectively.

\section{Authors' contributions}

All authors read and approved the final manuscript. AJS and PACH conceived and planned the study. BJ, RM, KKG, SMK, HHMD, and MAvdW performed the bioinformatic analyses. DH grew the cell lines and prepared the RNA-Seq libraries. BJ, RM, PACH, AJS, and SMK prepared the manuscript.

\section{Funding}

This work was supported by NIH grant HG006696 to AJS.

\section{Availability of data and materials}

The raw and processed RNA-Seq data for $165 \mathrm{LCL}$ samples have been deposited in the NCBI GEO database under accession number GSE92521 [64]. The 131 WB STAR-aligned BAM files (freeze 2) are submitted to the European Genome-phenome Archive (EGA) under study EGAS00001001077 and dataset accession number EGAD00001003937 [65]. The phased/imputed SNV data are part of the Genome of the Netherlands (GoNL) Project with EGA accession number EGAS00001000644 [66].

\section{Ethics approval and consent to participate}

Not applicable.

\section{Consent for publication}

The individuals in the GoNL study were participants from one of four biobanks: LifeLines-DEEP (LLD), Leiden Longevity Study (LLS), Netherlands Twin Registry (NTR), and Rotterdam Study (RS). The generation of the whole blood (WB) RNA-Seq and genotype data is explained in [20,56, 57]. Each participant gave consent for the use of data in research projects, including research with genotypes, as part of the individual participating cohorts (NTR, $\mathrm{LL}, \mathrm{LLS}$, and $\mathrm{RS}$ ).

\section{Competing interests}

The authors declare that they have no competing interests.

\section{Author details}

'Department of Genetics and Genomic Sciences, Hess Center for Science and Medicine, Mount Sinai School of Medicine, 1470 Madison Avenue, Room 8-116, Box 1498, New York, NY 10029, USA. ²Department of Biomedical Data Sciences, Leiden University Medical Center, Einthovenweg 20, 2333 ZC 
Leiden, the Netherlands. ${ }^{3}$ GenomeScan B.V., Plesmanlaan 1D, 2333 BZ Leiden, the Netherlands. ${ }^{4}$ Department of Human Genetics, Leiden University Medical Center, Leiden, the Netherlands. ${ }^{5}$ Department of Epidemiology and Biostatistics, VU University Medical Center, Amsterdam, the Netherlands. ${ }^{6}$ Department of Genetics, University Medical Center Groningen, Groningen, the Netherlands. ${ }^{7}$ Department of Internal Medicine, Erasmus MC, Rotterdam, the Netherlands. ${ }^{8}$ Department of Psychiatry, VU University Medical Center, Neuroscience Campus Amsterdam, Amsterdam, the Netherlands. ${ }^{9} \mathrm{C} e n t r e$ for Molecular and Biomolecular Informatics, Radboud Institute for Molecular Life Sciences, Radboud University Medical Center, Nijmegen, the Netherlands.

\section{Received: 9 November 2018 Accepted: 7 June 2019}

Published online: 24 June 2019

\section{References}

1. Haig D. Altercation of generations: genetic conflicts of pregnancy. Am J Reprod Immunol. 1996;35(3):226-32

2. Moore GE, Oakey R. The role of imprinted genes in humans. Genome Biol. 2011;12(3):106.

3. Bartolomei MS, Ferguson-Smith AC. Mammalian genomic imprinting. Cold Spring Harb Perspect Biol. 2011;3(7) Available from: https://doi.org/10.1101/ cshperspect.a002592

4. Azzi S, Rossignol S, Steunou V, Sas T, Thibaud N, Danton F, et al. Multilocus methylation analysis in a large cohort of 11p15-related foetal growth disorders (Russell Silver and Beckwith Wiedemann syndromes) reveals simultaneous loss of methylation at paternal and maternal imprinted loci. Hum Mol Genet. 2009;18(24):4724-33.

5. Nicholls RD, Saitoh S, Horsthemke B. Imprinting in Prader-Willi and Angelman syndromes. Trends Genet. 1998;14(5):194-200.

6. Mackay DJG, Callaway JLA, Marks SM, White HE, Acerini CL, Boonen SE, et al. Hypomethylation of multiple imprinted loci in individuals with transient neonatal diabetes is associated with mutations in ZFP57. Nat Genet. 2008; 40(8):949-51.

7. Steenman MJ, Rainier S, Dobry CJ, Grundy P, Horon IL, Feinberg AP. Loss of imprinting of IGF2 is linked to reduced expression and abnormal methylation of H19 in Wilms' tumour. Nat Genet. 1994;7(3):433-9.

8. Kaneda A, Feinberg AP. Loss of imprinting of IGF2: a common epigenetic modifier of intestinal tumor risk. Cancer Res. 2005;65(24):11236-40.

9. Piskol R, Ramaswami G, Li JB. Reliable identification of genomic variants from RNA-seq data. Am J Hum Genet. 2013;93(4):641-51.

10. Castel SE, Levy-Moonshine A, Mohammadi P, Banks E, Lappalainen T. Tools and best practices for data processing in allelic expression analysis. Genome Biol. 2015;16:195.

11. Baran Y, Subramaniam M, Biton A, Tukiainen T, Tsang EK, Rivas MA, et al. The landscape of genomic imprinting across diverse adult human tissues. Genome Res. 2015;25(7):927-36.

12. Babak T, DeVeale B, Tsang EK, Zhou Y, Li X, Smith KS, et al. Genetic conflict reflected in tissue-specific maps of genomic imprinting in human and mouse. Nat Genet. 2015;47(5):544-9.

13. Wang X, Sun Q, McGrath SD, Mardis ER, Soloway PD, Clark AG. Transcriptome-wide identification of novel imprinted genes in neonatal mouse brain. PLoS One. 2008;3(12):e3839.

14. Wang $X$, Soloway PD, Clark AG. A survey for novel imprinted genes in the mouse placenta by mRNA-seq. Genetics. 2011;189(1):109-22.

15. DeVeale B, van der Kooy D, Babak T. Critical evaluation of imprinted gene expression by RNA-Seq: a new perspective. PLoS Genet. 2012;8(3):e1002600.

16. Morcos L, Ge B, Koka V, Lam KCL, Pokholok DK, Gunderson KL, et al. Genome-wide assessment of imprinted expression in human cells. Genome Biol. 2011;12(3):R25.

17. Metsalu T, Viltrop T, Tiirats A, Rajashekar B, Reimann E, Kõks S, et al. Using RNA sequencing for identifying gene imprinting and random monoallelic expression in human placenta. Epigenetics. 2014;9(10):1397-409.

18. Mozaffari SV, Stein MM, Magnaye KM, Nicolae DL, Ober C. Parent of origin gene expression in a founder population identifies two new candidate imprinted genes at known imprinted regions. PLoS One. 2018;13(9):e0203906.

19. Zink F, Magnusdottir DN, Magnusson OT, Walker NJ, Morris TJ, Sigurdsson A, et al. Insights into imprinting from parent-of-origin phased methylomes and transcriptomes. Nat Genet. 2018;50(11):1542-52.

20. Genome of the Netherlands Consortium. Whole-genome sequence variation, population structure and demographic history of the Dutch population. Nat Genet. 2014;46(8):818-25.
21. Proudhon C, Bourc'his D. Identification and resolution of artifacts in the interpretation of imprinted gene expression. Brief Funct Genomics. 2010; 9(5-6):374-84.

22. Sanchez-Delgado M, Martin-Trujillo A, Tayama C, Vidal E, Esteller M, IglesiasPlatas I, et al. Absence of maternal methylation in biparental hydatidiform moles from women with NLRP7 maternal-effect mutations reveals widespread placenta-specific imprinting. PLoS Genet. 2015;11(11):e1005644.

23. Sanchez-Delgado M, Court F, Vidal E, Medrano J, Monteagudo-Sánchez A, Martin-Trujillo A, et al. Human oocyte-derived methylation differences persist in the placenta revealing widespread transient imprinting. PLoS Genet. 2016;12(11):e1006427.

24. Court F, Tayama C, Romanelli V, Martin-Trujillo A, Iglesias-Platas I, Okamura $\mathrm{K}$, et al. Genome-wide parent-of-origin DNA methylation analysis reveals the intricacies of human imprinting and suggests a germline methylationindependent mechanism of establishment. Genome Res. 2014;24(4):554-69.

25. Joshi RS, Garg P, Zaitlen N, Lappalainen T, Watson CT, Azam N, et al. DNA methylation profiling of uniparental disomy subjects provides a map of parental epigenetic bias in the human genome. Am J Hum Genet. 2016; 99(3):555-66.

26. Smilinich NJ, Day CD, Fitzpatrick GV, Caldwell GM, Lossie AC, Cooper PR, et al. A maternally methylated CpG island in KvLQT1 is associated with an antisense paternal transcript and loss of imprinting in Beckwith-Wiedemann syndrome. Proc Natl Acad Sci U S A. 1999;96(14):8064-9.

27. Stelzer Y, Shivalila CS, Soldner F, Markoulaki S, Jaenisch R. Tracing dynamic changes of DNA methylation at single-cell resolution. Cell. 2015;163(1):218-29.

28. Wood AJ, Schulz R, Woodfine K, Koltowska K, Beechey CV, Peters J, et al. Regulation of alternative polyadenylation by genomic imprinting. Genes Dev. 2008;22(9):1141-6.

29. Wood AJ, Roberts RG, Monk D, Moore GE, Schulz R, Oakey RJ. A screen for retrotransposed imprinted genes reveals an association between $X$ chromosome homology and maternal germ-line methylation. PLoS Genet. 2007;3(2):e20.

30. Nakabayashi K, Trujillo AM, Tayama C, Camprubi C, Yoshida W, Lapunzina P, et al. Methylation screening of reciprocal genome-wide UPDs identifies novel human-specific imprinted genes. Hum Mol Genet. 2011;20(16):3188-97.

31. Court F, Camprubi C, Garcia CV, Guillaumet-Adkins A, Sparago A, Seruggia $D$, et al. The PEG13-DMR and brain-specific enhancers dictate imprinted expression within the $8 q 24$ intellectual disability risk locus. Epigenetics Chromatin. 2014;7(1):5

32. Zylka MJ, Shearman LP, Weaver DR, Reppert SM. Three period homologs in mammals: differential light responses in the suprachiasmatic circadian clock and oscillating transcripts outside of brain. Neuron. 1998;20(6):1103-10.

33. Delaunay F, Thisse C, Marchand O, Laudet V, Thisse B. An inherited functional circadian clock in zebrafish embryos. Science. 2000;289(5477): 297-300.

34. Zhang L, Hirano A, Hsu P-K, Jones CR, Sakai N, Okuro M, et al. A PERIOD3 variant causes a circadian phenotype and is associated with a seasonal mood trait. Proc Natl Acad Sci U S A. 2016;113(11):E1536-44.

35. Monk D, Bentley L, Beechey C, Hitchins M, Peters J, Preece MA, et al. Characterisation of the growth regulating gene IMP3, a candidate for SilverRussell syndrome. J Med Genet. 2002;39(8):575-81.

36. Nielsen J, Christiansen J, Lykke-Andersen J, Johnsen AH, Wewer UM, Nielsen FC. A family of insulin-like growth factor II mRNA-binding proteins represses translation in late development. Mol Cell Biol. 1999;19(2):1262-70.

37. Bonthuis PJ, Huang W-C, Stacher Hörndli CN, Ferris E, Cheng T, Gregg C. Noncanonical genomic imprinting effects in offspring. Cell Rep. 2015;12(6): 979-91.

38. Edwards CA, Ferguson-Smith AC. Mechanisms regulating imprinted genes in clusters. Curr Opin Cell Biol. 2007;19(3):281-9.

39. Davies W, Isles A, Smith R, Karunadasa D, Burrmann D, Humby T, et al. XIr3b is a new imprinted candidate for $X$-linked parent-of-origin effects on cognitive function in mice. Nat Genet. 2005;37(6):625-9.

40. Raefski AS, O'Neill MJ. Identification of a cluster of X-linked imprinted genes in mice. Nat Genet. 2005;37(6):620-4.

41. Skuse DH, James RS, Bishop DV, Coppin B, Dalton P, Aamodt-Leeper G, et al Evidence from Turner's syndrome of an imprinted X-linked locus affecting cognitive function. Nature. 1997;387(6634):705-8.

42. Kosaki K, Kosaki R, Craigen WJ, Matsuo N. Isoform-specific imprinting of the human PEG1/MEST gene. Am J Hum Genet. 2000;66(1):309-12.

43. $\mathrm{Vu} T H$, Hoffman AR. Imprinting of the Angelman syndrome gene, UBE3A, is restricted to brain. Nat Genet. 1997;17(1):12-3. 
44. Valleley EM, Cordery SF, Bonthron DT. Tissue-specific imprinting of the ZAC/ PLAGL1 tumour suppressor gene results from variable utilization of monoallelic and biallelic promoters. Hum Mol Genet. 2007;16(8):972-81.

45. Kayashima T, Yamasaki K, Yamada T, Sakai H, Miwa N, Ohta T, et al. The novel imprinted carboxypeptidase A4 gene ( CPA4) in the 7q32 imprinting domain. Hum Genet. 2003;112(3):220-6.

46. Barlow DP. Gametic imprinting in mammals. Science. 1995;270(5242):1610-3.

47. Purcell S, Neale B, Todd-Brown K, Thomas L, Ferreira MAR, Bender D, et al. PLINK: a tool set for whole-genome association and population-based linkage analyses. Am J Hum Genet. 2007:81(3):559-75.

48. Chang CC, Chow CC, Tellier LC, Vattikuti S, Purcell SM, Lee JJ. Second-generation PLINK: rising to the challenge of larger and richer datasets. Gigascience. 2015;4:7.

49. Danecek P, Auton A, Abecasis G, Albers CA, Banks E, DePristo MA, et al. The variant call format and VCFtools. Bioinformatics. 2011;27(15):2156-8.

50. Browning SR, Browning BL. Rapid and accurate haplotype phasing and missing-data inference for whole-genome association studies by use of localized haplotype clustering. Am J Hum Genet. 2007;81 (5):1084-97.

51. Bolger AM, Lohse M, Usadel B. Trimmomatic: a flexible trimmer for Illumina sequence data. Bioinformatics. 2014;30(15):2114-20.

52. Dobin A, Davis CA, Schlesinger F, Drenkow J, Zaleski C, Jha S, et al. STAR: ultrafast universal RNA-seq aligner. Bioinformatics. 2013;29(1):15-21.

53. van de Geijn B, McVicker G, Gilad Y, Pritchard JK. WASP: allele-specific software for robust molecular quantitative trait locus discovery. Nat Methods. 2015:12(11):1061-3.

54. Conrad DF, Pinto D, Redon R, Feuk L, Gokcumen O, Zhang Y, et al. Origins and functional impact of copy number variation in the human genome. Nature. 2010;464(7289):704-12.

55. Zhernakova DV, Deelen P, Vermaat $M$, van Iterson M, van Galen M, Arindrarto $W$, et al. Identification of context-dependent expression quantitative trait loci in whole blood. Nat Genet. 2017;49(1):139-45.

56. Bonder MJ, Luijk R, Zhernakova DV, Moed M, Deelen P, Vermaat M, et al. Disease variants alter transcription factor levels and methylation of their binding sites. Nat Genet. 2017;49(1):131-8.

57. Browning BL, Yu Z. Simultaneous genotype calling and haplotype phasing improves genotype accuracy and reduces false-positive associations for genome-wide association studies. Am J Hum Genet. 2009;85(6):847-61.

58. Martin M. Cutadapt removes adapter sequences from high-throughput sequencing reads. EMBnet.journal. 2011;17(1):10-2.

59. Joshi NA, Fass JN, et al. Sickle: a sliding-window, adaptive, quality-based trimming tool for FastQ files (Version 1.33) [Software]. 2011.

60. McKenna A, Hanna M, Banks E, Sivachenko A, Cibulskis K, Kernytsky A, et al The Genome Analysis Toolkit: a MapReduce framework for analyzing nextgeneration DNA sequencing data. Genome Res. 2010;20(9):1297-303.

61. Sanna CR, Li W-H, Zhang L. Overlapping genes in the human and mouse genomes. BMC Genomics. 2008;9:169.

62. van de Wiel MA, Neerincx M, Buffart TE, Sie D, Verheul HMW. ShrinkBayes: a versatile R-package for analysis of count-based sequencing data in complex study designs. BMC Bioinformatics. 2014;15:116.

63. Rue H, Martino S, Chopin N. Approximate Bayesian inference for latent Gaussian models by using integrated nested Laplace approximations. J R Stat Soc Ser B Stat Methodol. 2009;71(2):319-92.

64. Sharp AJ, Jadhav B. Analysis of parent-of-origin bias in gene expression levels [Internet]. Dataset accession GSE92521. Gene Expression Omnibus (GEO); 2018. Available from: https://www.ncbi.nlm.nih.gov/geo/query/acc. cgi?acc $=$ GSE92521

65. BIOS Consortium (Additional file 22). BBMRI BIOS Project (Freeze 2) imprinting analysis, 131 whole blood aligned BAM files [Internet]. Dataset accession EGAD00001003937 ; Study accession EGAS00001001077. European Genome-phenome Archive (EGA); Available from: https://www. ebi.ac.uk/ega/studies/EGAS00001001077

66. The Genome of the Netherlands (GoNL) (Additional file 23). Phased/imputed SNV dataset [Internet]. Dataset accession EGAD00001000744; Study accession EGAS00001000644. European Genome-phenome Archive (EGA); Available from: https://www.ebi.ac.uk/ega/datasets/EGAD00001000744

67. Barbosa M, Joshi RS, Garg P, Martin-Trujillo A, Patel N, Jadhav B, et al. Identification of rare de novo epigenetic variations in congenital disorders. Nat Commun. 2018;9(1):2064.

\section{Publisher's Note}

Springer Nature remains neutral with regard to jurisdictional claims in published maps and institutional affiliations.

Ready to submit your research? Choose BMC and benefit from:

- fast, convenient online submission

- thorough peer review by experienced researchers in your field

- rapid publication on acceptance

- support for research data, including large and complex data types

- gold Open Access which fosters wider collaboration and increased citations

- maximum visibility for your research: over $100 \mathrm{M}$ website views per year

At $\mathrm{BMC}$, research is always in progress.

Learn more biomedcentral.com/submissions 